\title{
Yangian of the Queer Lie Superalgebra
}

\section{Maxim Nazarov}

Department of Mathematics, University of York, York YO1 5DD, England.

E-mail: mln1@york.ac.uk

\begin{abstract}
Consider the complex matrix Lie superalgebra $\mathfrak{g l}_{N \mid N}$ with the standard generators $E_{i j}$ where $i, j= \pm 1, \ldots, \pm N$. Define an involutory automorphism $\eta$ of $\mathfrak{g l}_{N \mid N}$ by $\eta\left(E_{i j}\right)=E_{-i,-j}$. The twisted polynomial current Lie superalgebra

$$
\mathfrak{g}=\left\{X(u) \in \mathfrak{g l}_{N \mid N}[u]: \eta(X(u))=X(-u)\right\}
$$
\end{abstract}

has a natural Lie co-superalgebra structure. We quantise the universal enveloping algebra $\mathrm{U}(\mathfrak{g})$ as a co-Poisson Hopf superalgebra. For the quantised algebra we give a description of the centre, and construct the double in the sense of Drinfeld. We also construct a wide class of irreducible representations of the quantised algebra.

\section{Introduction}

In this article we will work with certain Lie superalgebras $[\mathrm{K}]$ over the complex field $\mathbb{C}$. Their universal enveloping algebras are $\mathbb{Z}_{2}$-graded associative unital algebras, and we will always keep to the following convention. Let $\mathrm{A}$ and $\mathrm{B}$ be any two associative complex $\mathbb{Z}_{2}$-graded algebras. Their tensor product $\mathrm{A} \otimes \mathrm{B}$ will be a $\mathbb{Z}_{2}$-graded algebra such that for any homogeneous $X, X^{\prime} \in \mathrm{A}$ and $Y, Y^{\prime} \in \mathrm{B}$

$$
\begin{aligned}
(X \otimes Y)\left(X^{\prime} \otimes Y^{\prime}\right) & =X X^{\prime} \otimes Y Y^{\prime} \cdot(-1)^{\operatorname{deg} X^{\prime} \operatorname{deg} Y}, \\
\operatorname{deg}(X \otimes Y) & =\operatorname{deg} X+\operatorname{deg} Y .
\end{aligned}
$$

Throughout this article we will denote by $\theta$ the isomorphism $\mathrm{A} \otimes \mathrm{B} \rightarrow \mathrm{B} \otimes \mathrm{A}$ defined by

$$
X \otimes Y \mapsto Y \otimes X \cdot(-1)^{\operatorname{deg} X \operatorname{deg} Y} .
$$

If the algebra $\mathrm{A}$ is unital denote by $\iota_{p}$ its embedding into the tensor product $\mathrm{A}^{\otimes n}$ as the $p$-th tensor factor:

$$
\iota_{p}(X)=1^{\otimes(p-1)} \otimes X \otimes 1^{\otimes(n-p)}, \quad 1 \leqslant p \leqslant n .
$$

We will also use various embeddings of the algebra $\mathrm{A}^{\otimes m}$ into $\mathrm{A}^{\otimes n}$ for any $m \leqslant n$. For any choice of pairwise distinct indices $p_{1}, \ldots, p_{m} \in\{1, \ldots, n\}$ and an element $X \in \mathrm{A}^{\otimes m}$ of the form $X=X^{(1)} \otimes \ldots \otimes X^{(m)}$ we will denote

$$
X_{p_{1} \ldots p_{m}}=\iota_{p_{1}}\left(X^{(1)}\right) \ldots \iota_{p_{m}}\left(X^{(m)}\right) \in \mathrm{A}^{\otimes n} .
$$

Let $\mathfrak{a}$ be an arbitrary finite-dimensional Lie superalgebra. Then consider the polynomial current Lie superalgebra $\mathfrak{a}[u]$. It consists of the polynomial functions of a complex variable $u$ valued in $\mathfrak{a}$. For any two such functions their supercommutator in $\mathfrak{a}[u]$ is determined pointwise. Let $K \in \mathfrak{a}^{\otimes 2}$ be an $\mathfrak{a}$-invariant element: we have the equality $\left[X_{1}+X_{2}, K\right]=0$ in $\mathfrak{a}^{\otimes 2}$ for any $X \in \mathfrak{a}$. Here $\mathfrak{a}$ is regarded 
as a subspace in the enveloping algebra $U(\mathfrak{a})$ and the square brackets stand for the supercommutator. Also suppose that $K$ is of $\mathbb{Z}_{2}$-degree zero and symmetric: $K_{12}=K_{21}$. Then the rational function

$$
r(u, v)=\frac{K}{u-v}
$$

of two complex variables $u, v$ satisfies the classical Yang-Baxter equation for $\mathfrak{a}[u]$ :

$$
\left[r_{12}(u, v), r_{13}(u, w)\right]+\left[r_{12}(u, v), r_{23}(v, w)\right]+\left[r_{13}(u, w), r_{23}(v, w)\right]=0
$$

in $\mathfrak{a}^{\otimes 3}$. This can be verified by momentary calculation. Furthermore, the function (1.1) is antisymmetric:

$$
r_{12}(u, v)+r_{21}(v, u)=0 .
$$

Therefore the co-supercommutator $\varphi: \mathfrak{a}[u] \rightarrow \mathfrak{a}[u]^{\otimes 2}=\mathfrak{a}^{\otimes 2}[u, v]$ can be defined by

$$
\varphi(X(u))=\left[X_{1}(u)+X_{2}(v), r(u, v)\right] .
$$

This definition makes $\mathfrak{a}[u]$ into a Lie bi-superalgebra. In particular, if $\mathfrak{a}$ is a simple Lie algebra and $K$ is the Casimir element, one gets a natural Lie bialgebra structure on $\mathfrak{a}[u]$. It gives rise to a natural co-Poisson structure on the universal enveloping algebra $\mathrm{U}(\mathfrak{a}[u])$, which is a co-commutative Hopf algebra by definition. The more general case of a simple Lie superalgebra $\mathfrak{a}$ was considered in [LS].

Now consider the queer Lie superalgebra $\mathfrak{q}_{N}$. This is the most interesting superanalogue of the general linear Lie algebra $\mathfrak{g l}_{N}$, see for instance [S2]. We will realise $\mathfrak{q}_{N}$ as a subalgebra in the general linear Lie superalgebra $\mathfrak{g l}_{N \mid N}$. Let the indices $i, j$ run through $\pm 1, \ldots, \pm N$. We will always write $\bar{\imath}=0$ if $i>0$ and $\bar{\imath}=1$ if $i<0$. Consider the $\mathbb{Z}_{2}$-graded vector space $\mathbb{C}^{N \mid N}$. Let $e_{i} \in \mathbb{C}^{N \mid N}$ be an element of the standard basis. The $\mathbb{Z}_{2}$-gradation on $\mathbb{C}^{N \mid N}$ is defined so that $\operatorname{deg} e_{i}=\bar{\imath}$. Let $E_{i j} \in \operatorname{End}\left(\mathbb{C}^{N \mid N}\right)$ be the standard matrix units. The algebra End $\left(\mathbb{C}^{N \mid N}\right)$ is $\mathbb{Z}_{2}$-graded so that $\operatorname{deg} E_{i j}=\bar{\imath}+\bar{\jmath}$. We will also regard $E_{i j}$ as generators of the complex Lie superalgebra $\mathfrak{g l}_{N \mid N}$. The queer classical Lie superalgebra $\mathfrak{q}_{N}$ is the fixed point subalgebra in $\mathfrak{g l}_{N \mid N}$ with respect to the involutive automorphism

$$
\eta: E_{i j} \mapsto E_{-i,-j}
$$

The queerness of $\mathfrak{q}_{N}$ reveals itself in that all the symmetric $\mathfrak{q}_{N}$-invariants in $\mathfrak{q}_{N}^{\otimes 2}$ of $\mathbb{Z}_{2}$-degree zero are trivial: for $\mathfrak{a}=\mathfrak{q}_{N}$ we always have $K \in \mathbb{C} \cdot E^{\otimes 2}$ where

$$
E=E_{11}+E_{-1,-1}+\ldots+E_{N N}+E_{-N,-N} .
$$

Hence in this case the co-supercommutator (1.3) vanishes and there is no natural Lie bi-superalgebra structure on $\mathfrak{q}_{N}[u]$. However such a structure can be defined, in compensation, on the twisted polynomial current Lie superalgebra

$$
\mathfrak{g}=\left\{X(u) \in \mathfrak{g l}_{N \mid N}[u]: \eta(X(u))=X(-u)\right\} .
$$

Our definition is based on the following general scheme [A1,A2,FR]. Let $\mathfrak{a}, K$ be arbitrary as above and $\omega$ be an automorphism of the Lie superalgebra $\mathfrak{a}$ of finite order $n$. Let $\zeta$ be a primitive $n$-th root of unity. Generalising (1.1) put

$$
r(u, v)=\sum_{m \in \mathbb{Z}_{n}} \frac{\mathrm{id} \otimes \omega^{m}(K)}{u-\zeta^{m} v} .
$$


Proposition 1.1. Suppose that $\omega^{\otimes 2}(K)=\zeta K$. Then the function (1.7) is antisymmetric and obeys the classical Yang-Baxter equation (1.2).

Proof. The function $r(u, v)$ determined by (1.1) satisfies the equation (1.2). Let us apply to the left-hand side of (1.2) with that $r(u, v)$ the operator id $\otimes \omega^{k} \otimes \omega^{l}$ in $\mathfrak{a}^{\otimes 3}$ and substitute $\zeta^{k} v, \zeta^{l} w$ for $v, w$ respectively. Taking then the sum over $k, l \in$ $\mathbb{Z}_{n}$ and using $\omega^{\otimes 2}(K)=\zeta K$, we will obtain the left-hand side of (1.2) with the function $r(u, v)$ determined by (1.7). For the latter function $r(u, v)$ we also have

$$
\begin{aligned}
r_{21}(v, u) & =\sum_{m \in \mathbb{Z}_{n}} \frac{\omega^{m} \otimes \mathrm{id}\left(K_{21}\right)}{v-\zeta^{m} u}=\sum_{m \in \mathbb{Z}_{n}} \frac{\omega^{m} \otimes \mathrm{id}(K)}{v-\zeta^{m} u} \\
& =\sum_{m \in \mathbb{Z}_{n}} \frac{\mathrm{id} \otimes \omega^{-m}(K)}{\zeta^{-m} v-u}=-r(u, v)
\end{aligned}
$$

Note that for a simple Lie algebra $\mathfrak{a}$ always $\omega^{\otimes 2}(K)=K$, and in compliance with $[\mathrm{BD}]$ this construction does not give any new solutions of (1.2). Let $\mathfrak{a}$ be the Lie superalgebra $\mathfrak{g l}_{N \mid N}$ and $\omega$ be the involutive automorphism (1.4). The element

$$
P=\sum_{i j} E_{i j} \otimes E_{j i} \cdot(-1)^{\bar{\jmath}}
$$

of $\mathfrak{g l}_{N \mid N}^{\otimes 2}$ is symmetric and $\mathfrak{g l}_{N \mid N}$-invariant. Moreover, we have $\eta^{\otimes 2}(P)=-P$. Due to Proposition 1.1 by setting $K=P$ in (1.7) we get an antisymmetric solution of the Yang-Baxter equation (1.2). Therefore (1.3) defines a co-supercommutator $\varphi: \mathfrak{g} \rightarrow \mathfrak{g}^{\otimes 2}$. Thus we obtain a Lie bi-superalgebra structure on $\mathfrak{g}$.

For any simple finite-dimensional Lie algebra $\mathfrak{a}$, quantisation of the co-Poisson Hopf algebra $\mathrm{U}(\mathfrak{a}[u])$ was described in [D1]. The quantised Hopf algebra is denoted by $Y(\mathfrak{a})$ and called the Yangian of the Lie algebra $\mathfrak{a}$. The algebra $Y(\mathfrak{a})$ contains the universal enveloping algebra $\mathrm{U}(\mathfrak{a})$ as a subalgebra. However, the case $\mathfrak{a}=\mathfrak{s l}_{N}$ is exceptional since only for $\mathfrak{a}=\mathfrak{s l}_{N}$ there exists a homomorphism $\mathrm{Y}(\mathfrak{a}) \rightarrow \mathrm{U}(\mathfrak{a})$ identical on the subalgebra $U(\mathfrak{a})$, see [D1, Theorem 9]. There is also a Hopf algebra $\mathrm{Y}\left(\mathfrak{g l}_{N}\right)$, which is a quantisation of the co-Poisson Hopf algebra $\mathrm{U}\left(\mathfrak{g l}_{N}[u]\right)$. Again, the algebra $\mathrm{Y}\left(\mathfrak{g l}_{N}\right)$ contains the enveloping algebra $\mathrm{U}\left(\mathfrak{g l}_{N}\right)$ as a subalgebra, and admits a homomorphism $\mathrm{Y}\left(\mathfrak{g l}_{N}\right) \rightarrow \mathrm{U}\left(\mathfrak{g l}_{N}\right)$ identical on $\mathrm{U}\left(\mathfrak{g l}_{N}\right)$. Moreover, the algebra $\mathrm{Y}\left(\mathfrak{g l}_{N}\right)$ can be defined entirely in terms of the classical representation theory [O1]. For further details on the Yangian $\mathrm{Y}\left(\mathfrak{g l}_{N}\right)$ see $[\mathrm{MNO}]$ and references therein.

The main aim of this article is to define the Yangian of the Lie superalgebra $\mathfrak{q}_{N}$. It cannot be defined as a quantisation of the enveloping algbra $\mathrm{U}\left(\mathfrak{q}_{N}[u]\right)$, because the latter Hopf superalgebra has no natural co-Poisson structure. Instead of $\mathfrak{q}_{N}[u]$ we will consider the twisted polynomial current Lie superalgebra $\mathfrak{g}$. In Section 2 we define a certain Hopf superalgebra $\mathrm{Y}\left(\mathfrak{q}_{N}, h\right)$ over the field $\mathbb{C}[[h]]$ of the formal power series in $h$. The quotient $\mathrm{Y}\left(\mathfrak{q}_{N}, h\right) / h \mathrm{Y}\left(\mathfrak{q}_{N}, h\right)$ is isomorphic to $\mathrm{U}(\mathfrak{g})$ as a co-Poisson Hopf superalgebra. All specialisations of $\mathrm{Y}\left(\mathfrak{q}_{N}, h\right)$ at $h \in \mathbb{C} \backslash\{0\}$ are isomorphic to each other as Hopf superalgebras. The specialisation at $h=1$ will be denoted by $\mathrm{Y}\left(\mathfrak{q}_{N}\right)$ and called the Yangian of Lie superalgebra $\mathfrak{q}_{N}$. Similarly to the Yangian $\mathrm{Y}\left(\mathfrak{g l}_{N}\right)$, the algebra $\mathrm{Y}\left(\mathfrak{q}_{N}\right)$ contains the enveloping algebra $\mathrm{U}\left(\mathfrak{q}_{N}\right)$ as a subalgebra, and admits a homomorphism $\mathrm{Y}\left(\mathfrak{q}_{N}\right) \rightarrow \mathrm{U}\left(\mathfrak{q}_{N}\right)$ identical on $\mathrm{U}\left(\mathfrak{q}_{N}\right)$. In Section 3 we describe the centre of the $\mathbb{Z}_{2}$-graded algebra $\mathrm{Y}\left(\mathfrak{q}_{N}\right)$. In Section 4 we construct the double of this Yangian in the sense of [D3]. In Section 5 we study an analogue for $\mathrm{Y}\left(\mathfrak{q}_{N}\right)$ of the Drinfeld functor [D2] for the Yangian $\mathrm{Y}\left(\mathfrak{g l}_{N}\right)$. 


\section{Definition of the Yangian}

In this section we introduce the Yangian of the Lie superalgebra $\mathfrak{q}_{N}$. This is a complex associative unital $\mathbb{Z}_{2}$-graded algebra $\mathrm{Y}\left(\mathfrak{q}_{N}\right)$ with the countable set of generators $T_{i j}^{(s)}$ where $s=1,2, \ldots$ and $i, j= \pm 1, \ldots, \pm N$. The $\mathbb{Z}_{2}$-gradation on the algebra $\mathrm{Y}\left(\mathfrak{q}_{N}\right)$ is determined by setting $\operatorname{deg} T_{i j}^{(s)}=\bar{\imath}+\bar{\jmath}$ for $s \geqslant 1$. To write down defining relations for these generators we will employ the formal series

$$
T_{i j}(u)=\delta_{i j} \cdot 1+T_{i j}^{(1)} u^{-1}+T_{i j}^{(2)} u^{-2}+\ldots
$$

from $\mathrm{Y}\left(\mathfrak{q}_{N}\right)\left[\left[u^{-1}\right]\right]$. Then for all possible indices $i, j$ and $k, l$ we have the relations

$$
\begin{gathered}
\left(u^{2}-v^{2}\right) \cdot\left[T_{i j}(u), T_{k l}(v)\right] \cdot(-1)^{\bar{\imath} \bar{k}+\bar{\imath} \bar{l}+\bar{k} \bar{l}}= \\
(u+v) \cdot\left(T_{k j}(u) T_{i l}(v)-T_{k j}(v) T_{i l}(u)\right)- \\
(u-v) \cdot\left(T_{-k, j}(u) T_{-i, l}(v)-T_{k,-j}(v) T_{i,-l}(u)\right) \cdot(-1)^{\bar{k}+\bar{l}}
\end{gathered}
$$

in $\mathrm{Y}\left(\mathfrak{q}_{N}\right)\left(\left(u^{-1}, v^{-1}\right)\right)$. The square brackets here stand for the supercommutator. Moreover, for all possible indices $i, j$ we impose the relations

$$
T_{i j}(-u)=T_{-i,-j}(u)
$$

We will also use the following matrix form of the relations (2.2). Regard $E_{i j}$ as elements of the algebra $\operatorname{End}\left(\mathbb{C}^{N \mid N}\right)$. Combine all the series (2.1) into the single element

$$
T(u)=\sum_{i j} E_{i j} \otimes T_{i j}(u)
$$

of the algebra $\operatorname{End}\left(\mathbb{C}^{N \mid N}\right) \otimes \mathrm{Y}\left(\mathfrak{q}_{N}\right)\left[\left[u^{-1}\right]\right]$. For any positive integer $n$ and each $s=1, \ldots, n$ we denote

$$
T_{s}(u)=\iota_{s} \otimes \operatorname{id}(T(u)) \in \operatorname{End}\left(\mathbb{C}^{N \mid N}\right)^{\otimes n} \otimes \mathrm{Y}\left(\mathfrak{q}_{N}\right)\left[\left[u^{-1}\right]\right] .
$$

Regard (1.8) as an element of the algebra $\operatorname{End}\left(\mathbb{C}^{N \mid N}\right)^{\otimes 2}$. Consider the element

$$
J=\sum_{i} E_{i,-i} \cdot(-1)^{\bar{\imath}}
$$

of the algebra End $\left(\mathbb{C}^{N \mid N}\right)$, it has $\mathbb{Z}_{2}$-degree one. Note that the supercommutant of this element in End $\left(\mathbb{C}^{N \mid N}\right)$ coincides with the image of the defining representation $\mathfrak{q}_{N} \rightarrow \operatorname{End}\left(\mathbb{C}^{N \mid N}\right)$. Introduce the rational function of two complex variables $u, v$

$$
\begin{gathered}
R(u, v)=1-\frac{P}{u-v}+\frac{P J_{1} J_{2}}{u+v}= \\
1-\sum_{i j} E_{i j} \otimes E_{j i} \cdot \frac{(-1)^{\bar{\jmath}}}{u-v}-\sum_{i j} E_{i j} \otimes E_{-j,-i} \cdot \frac{(-1)^{\bar{\jmath}}}{u+v}
\end{gathered}
$$

valued in the algebra End $\left(\mathbb{C}^{N \mid N}\right)^{\otimes 2}$. Then the relations $(2.2)$ can be rewritten as

$$
(R(u, v) \otimes 1) \cdot T_{1}(u) T_{2}(v)=T_{2}(v) T_{1}(u) \cdot(R(u, v) \otimes 1)
$$


Namely, after multiplying each side of $(2.7)$ by $u^{2}-v^{2}$ it becomes a relation in the algebra

$$
\operatorname{End}\left(\mathbb{C}^{N \mid N}\right)^{\otimes 2} \otimes \mathrm{Y}\left(\mathfrak{q}_{N}\right)\left(\left(u^{-1}, v^{-1}\right)\right)
$$

equivalent to the collection of all relations (2.2). Also note that the function (2.6) satisfies the quantum Yang-Baxter equation for the algebra $\operatorname{End}\left(\mathbb{C}^{N \mid N}\right)^{\otimes 3}(u, v, w)$

$$
R_{12}(u, v) R_{13}(u, w) R_{23}(v, w)=R_{23}(v, w) R_{13}(u, w) R_{12}(u, v) .
$$

Furthermore, consider (1.4) as an automorphism of the algebra End $\left(\mathbb{C}^{N \mid N}\right)$. The collection of all relations (2.3) is equivalent to the single equation

$$
\eta \otimes \mathrm{id}(T(u))=T(-u) \text {. }
$$

Observe that by the definition (2.6) of $R(u, v)$ we also have in $\operatorname{End}\left(\mathbb{C}^{N \mid N}\right)^{\otimes 2}(u, v)$

$$
\begin{aligned}
& \eta \otimes \mathrm{id}(R(u, v))=R(-u, v), \\
& \mathrm{id} \otimes \eta(R(u, v))=R(u,-v) .
\end{aligned}
$$

We call the function (2.6) the rational $R$-matrix for the Lie superalgebra $\mathfrak{q}_{N}$.

For any $i, j$ put $F_{i j}=E_{i j}+E_{-i,-j}$. Then we have the equality $\eta\left(F_{i j}\right)=F_{i j}$ in $\operatorname{End}\left(\mathbb{C}^{N \mid N}\right)$. We will also regard $F_{i j}$ as generators of the universal enveloping algebra $\mathrm{U}\left(\mathfrak{q}_{N}\right)$. Due to $(2.2)$ there is a homomorphism

$$
\mathrm{Y}\left(\mathfrak{q}_{N}\right) \rightarrow \mathrm{U}\left(\mathfrak{q}_{N}\right): T_{i j}(u) \mapsto \delta_{i j}-F_{j i} u^{-1} \cdot(-1)^{\bar{j}} .
$$

The relations (2.2),(2.3) now imply that the assignment

$$
F_{j i} \mapsto-T_{i j}^{(1)} \cdot(-1)^{\bar{\jmath}}
$$

defines embedding of $\mathbb{Z}_{2}$-graded associative unital algebras $\mathrm{U}\left(\mathfrak{q}_{N}\right) \rightarrow \mathrm{Y}\left(\mathfrak{q}_{N}\right)$. The fact that the homomorphism (2.13) has no kernel, is due to Theorem 2.3 below.

The homomorphism (2.12) is identical on the subalgebra $\mathrm{U}\left(\mathfrak{q}_{N}\right)$. It will be called the evaluation homomorphism for the algebra $\mathrm{Y}\left(\mathfrak{q}_{N}\right)$ and denoted by $\pi_{N}$. The element $T(u)$ of the algebra $\operatorname{End}\left(\mathbb{C}^{N \mid N}\right) \otimes \mathrm{Y}\left(\mathfrak{q}_{N}\right)\left[\left[u^{-1}\right]\right]$ is invertible, we put

$$
T(u)^{-1}=\sum_{i, j} E_{i j} \otimes \widetilde{T}_{i j}(u) .
$$

Then the relations $(2.7),(2.9)$ along with the identity

$$
R(u, v) R(-u,-v)=1-\frac{1}{(u-v)^{2}}-\frac{1}{(u+v)^{2}}
$$

imply that the assignment $T_{i j}(u) \mapsto \widetilde{T}_{i j}(-u)$ determines an automorphism of the algebra $\mathrm{Y}\left(\mathfrak{q}_{N}\right)$. This automorphism is evidently involutive.

We will use two different ascending $\mathbb{Z}$-filtrations on the algebra $\mathrm{Y}\left(\mathfrak{q}_{N}\right)$. They are obtained by assigning to the generator $T_{i j}^{(s)}$ the degree $s$ or $s-1$ respectively. The corresponding $\mathbb{Z}$-graded algebras will be denoted by gr $\mathrm{Y}\left(\mathfrak{q}_{N}\right)$ and $\operatorname{gr}{ }_{-} \mathrm{Y}\left(\mathfrak{q}_{N}\right)$. Let $G_{i j}^{(s)} \in \operatorname{gr}_{-} \mathrm{Y}\left(\mathfrak{q}_{N}\right)$ be the element corresponding to the generator $T_{i j}^{(s)} \in \mathrm{Y}\left(\mathfrak{q}_{N}\right)$. The algebra gr_Y $\left(\mathfrak{q}_{N}\right)$ inherits $\mathbb{Z}_{2}$-gradation from $\mathrm{Y}\left(\mathfrak{q}_{N}\right)$ such that $\operatorname{deg} G_{i j}^{(s)}=\bar{\imath}+\bar{\jmath}$.

Take the enveloping algebra $U(\mathfrak{g})$ of the twisted current Lie superalgebra (1.6). The algebra $U(\mathfrak{g})$ also has a natural $\mathbb{Z}_{2}$-gradation: the $\mathbb{Z}_{2}$-degree of the element

$$
F_{i j}^{(s)}=E_{i j} u^{s}+E_{-i,-j}(-u)^{s}
$$

equals $\bar{\imath}+\bar{\jmath}$ for any $s \geqslant 0$. We have the following easy observation. 
Proposition 2.1. The assignment for every $s \geqslant 0$

$$
F_{j i}^{(s)} \mapsto-G_{i j}^{(s+1)} \cdot(-1)^{\bar{\jmath}}
$$

determines a surjective homomorphism $\mathrm{U}(\mathfrak{g}) \rightarrow \operatorname{gr} \mathrm{Y}_{-}\left(\mathfrak{q}_{N}\right)$ of $\mathbb{Z}_{2}$-graded algebras.

Proof. The elements (2.15) generate the algebra $\mathrm{U}(\mathfrak{g})$. The defining relations for these generators can be written as

$$
\begin{gathered}
{\left[F_{j i}^{(s)}, F_{l k}^{(r)}\right]=\delta_{i l} F_{j k}^{(s+r)}-\delta_{k j} F_{l i}^{(s+r)} \cdot(-1)^{(\bar{\imath}+\bar{\jmath})(\bar{l}+\bar{k})}+} \\
\delta_{i,-l} F_{-j, k}^{(s+r)} \cdot(-1)^{s}-\delta_{-k, j} F_{l,-i}^{(s+r)} \cdot(-1)^{(\bar{\imath}+\bar{\jmath})(\bar{l}+\bar{k})+s}
\end{gathered}
$$

for all $r, s \geqslant 0$ and

$$
F_{-j,-i}^{(s)}=(-1)^{s} \cdot F_{j i}^{(s)}
$$

On the other hand, by $(2.2)$ we obtain the relations in the algebra $\operatorname{gr}_{-} \mathrm{Y}\left(\mathfrak{q}_{N}\right)$

$$
\begin{gathered}
(-1)^{\bar{\imath} \bar{k}+\bar{\imath} \bar{l}+\bar{k} \bar{l}} \cdot\left[G_{i j}^{(s)}, G_{k l}^{(r)}\right]=\delta_{k j} G_{i l}^{(s+r-1)}-\delta_{i l} G_{k j}^{(s+r-1)}+ \\
\delta_{k,-j} G_{-i, l}^{(s+r-1)} \cdot(-1)^{\bar{k}+\bar{l}+s}-\delta_{-i, l} G_{k,-j}^{(s+r-1)} \cdot(-1)^{\bar{k}+\bar{l}+s}
\end{gathered}
$$

for $r, s \geqslant 1$. Due to $(2.3)$

$$
G_{-i,-j}^{(s)}=(-1)^{s} \cdot G_{i j}^{(s)} .
$$

Comparison of these relations to (2.17) and (2.18) shows that (2.16) determines a homomorphism $\mathrm{U}(\mathfrak{g}) \rightarrow \mathrm{gr}_{-} \mathrm{Y}\left(\mathfrak{q}_{N}\right)$. This homomorphism is surjective and preserves $\mathbb{Z}_{2}$-gradation by definition

There is a natural Hopf superalgebra structure on $\mathrm{Y}\left(\mathfrak{q}_{N}\right)$. Due to $(2.7),(2.9)$ the comultiplication $\Delta: \mathrm{Y}\left(\mathfrak{q}_{N}\right) \rightarrow \mathrm{Y}\left(\mathfrak{q}_{N}\right) \otimes \mathrm{Y}\left(\mathfrak{q}_{N}\right)$ can be defined by

$$
T_{i j}(u) \mapsto \sum_{k} T_{i k}(u) \otimes T_{k j}(u) \cdot(-1)^{(\bar{\imath}+\bar{k})(\bar{\jmath}+\bar{k})}
$$

where the tensor product is taken over the subalgebra $\mathbb{C}\left[\left[u^{-1}\right]\right]$ in $\mathrm{Y}\left(\mathfrak{q}_{N}\right)\left[\left[u^{-1}\right]\right]$ and the index $k$ runs through $\pm 1, \ldots, \pm N$. The counit $\varepsilon: \mathrm{Y}\left(\mathfrak{q}_{N}\right) \rightarrow \mathbb{C}$ is defined so that $\varepsilon: T_{i j}^{(-s)} \mapsto 0$ for every $s \geqslant 1$. Then the assignment $T_{i j}(u) \mapsto \widetilde{T}_{i j}(u)$ determines the antipodal map $S: \mathrm{Y}\left(\mathfrak{q}_{N}\right) \rightarrow \mathrm{Y}\left(\mathfrak{q}_{N}\right)$. It is an antiautomorphism of the $\mathbb{Z}_{2}$-graded algebra $\mathrm{Y}\left(\mathfrak{q}_{N}\right)$. Note that $\mathrm{Y}\left(\mathfrak{q}_{N}\right)$ contains $\mathrm{U}\left(\mathfrak{q}_{N}\right)$ as a Hopf sub-superalgebra: by the definitions $(2.13)$ and (2.19) for any $F \in \mathfrak{q}_{N}$ we have

$$
\Delta(F)=F \otimes 1+1 \otimes F, \quad \varepsilon(F)=0, \quad S(F)=-F .
$$

The comultiplication (2.19) on the $\mathbb{Z}_{2}$-graded algebra $\mathrm{Y}\left(\mathfrak{q}_{N}\right)$ allows us to define for any $n=1,2, \ldots$ a representation $\mathrm{Y}\left(\mathfrak{q}_{N}\right) \rightarrow \operatorname{End}\left(\mathbb{C}^{N \mid N}\right)^{\otimes n}$ depending on $n$ arbitrary complex parameters $z_{1}, \ldots, z_{n}$. Indeed, by comparing (2.8),(2.10) to $(2.7),(2.9)$ respectively we obtain that for any $z \in \mathbb{C}$ the assignment

$$
\operatorname{End}\left(\mathbb{C}^{N \mid N}\right) \otimes \mathrm{Y}\left(\mathfrak{q}_{N}\right)\left[\left[u^{-1}\right]\right] \rightarrow \operatorname{End}\left(\mathbb{C}^{N \mid N}\right)^{\otimes 2}\left[\left[u^{-1}\right]\right]: T(u) \mapsto R(u, z)
$$


determines a representation $\mathrm{Y}\left(\mathfrak{q}_{N}\right) \rightarrow \operatorname{End}\left(\mathbb{C}^{N \mid N}\right)$. More explicitly, we have

$$
T_{i j}^{(s+1)} \mapsto-\left(E_{j i} z^{s}+E_{-j,-i}(-z)^{s}\right) \cdot(-1)^{\bar{\jmath}}, \quad s \geqslant 0 .
$$

When $z=0$ this representation $\mathrm{Y}\left(\mathfrak{q}_{N}\right)$ can be also obtained from the standard representation $\mathrm{U}\left(\mathfrak{q}_{N}\right) \rightarrow \operatorname{End}\left(\mathbb{C}^{N \mid N}\right)$ by virtue of the evaluation homomorphism (2.12). Now for any $z_{1}, \ldots, z_{n} \in \mathbb{C}$ take the tensor product of the representations (2.20) of the algebra $\mathrm{Y}\left(\mathfrak{q}_{N}\right)$ with $z=z_{1}, \ldots, z_{n}$. Due to (2.19) the respective homomorphism $\mathrm{Y}\left(\mathfrak{q}_{N}\right) \rightarrow$ End $\left(\mathbb{C}^{N \mid N}\right)^{\otimes n}$ is determined by the assignment

$$
\begin{gathered}
\operatorname{End}\left(\mathbb{C}^{N \mid N}\right) \otimes \mathrm{Y}\left(\mathfrak{q}_{N}\right)\left[\left[u^{-1}\right]\right] \rightarrow \operatorname{End}\left(\mathbb{C}^{N \mid N}\right)^{\otimes(n+1)}\left[\left[u^{-1}\right]\right]: \\
T(u) \mapsto R_{12}\left(u, z_{1}\right) \ldots R_{1, n+1}\left(u, z_{n}\right) .
\end{gathered}
$$

Proposition 2.2. Let the complex parameters $z_{1}, \ldots, z_{n}$ and positive integer $n$ vary. Then the kernels of all representations (2.22) of $\mathrm{Y}\left(\mathfrak{q}_{N}\right)$ have zero intersection.

Proof. Take any finite linear combination of the products $T_{i_{1} j_{1}}^{\left(s_{1}\right)} \ldots T_{i_{m} j_{m}}^{\left(s_{m}\right)} \in \mathrm{Y}\left(\mathfrak{q}_{N}\right)$ with certain complex coefficients $A_{i_{1} j_{1} \ldots i_{m} j_{m}}^{\left(s_{1} \ldots s_{m}\right)}$ where the indices $s_{1}, \ldots, s_{m} \geqslant 1$ and the number $m \geqslant 0$ may vary. Consider the image of this combination under the representation $\mathrm{Y}\left(\mathfrak{q}_{N}\right) \rightarrow \operatorname{End}\left(\mathbb{C}^{N \mid N}\right)^{\otimes n}$ determined by $(2.22)$; it depends on $z_{1}, \ldots, z_{n}$ polynomially. Take the terms of this polynomial which have the maximal total degree in $z_{1}, \ldots, z_{n}$. Let $A$ the sum of these terms and $d$ be their degree.

Consider the ascending $\mathbb{Z}$-filtration on algebra $\mathrm{Y}\left(\mathfrak{q}_{N}\right)$ where the generator $T_{i j}^{(s)}$ with $s \geqslant 1$ has degree $s-1$. Equip the tensor product $\mathrm{Y}\left(\mathfrak{q}_{N}\right)^{\otimes n}$ with the ascending $\mathbb{Z}$-filtration where the degree is the sum of the degrees on the tensor factors. Then by the definition (2.19) under the comultiplication $\mathrm{Y}\left(\mathfrak{q}_{N}\right) \rightarrow \mathrm{Y}\left(\mathfrak{q}_{N}\right)^{\otimes n}$ we have

$$
T_{i j}^{(s)} \mapsto \sum_{1 \leqslant r \leqslant n} 1^{\otimes(r-1)} \otimes T_{i j}^{(s)} \otimes 1^{\otimes(n-r)}+\text { lower degree terms }, \quad s \geqslant 1 .
$$

Therefore $A \in \operatorname{End}\left(\mathbb{C}^{N \mid N}\right)^{\otimes n}$ coincides with the image of the sum

$$
\sum_{s_{1}+\ldots+s_{m}=d+m} A_{i_{1} j_{1} \ldots i_{m} j_{m}}^{\left(s_{1} \ldots s_{m}\right)} F_{j_{1} i_{1}}^{\left(s_{1}-1\right)} \ldots F_{j_{m} i_{m}}^{\left(s_{m}-1\right)} \cdot(-1)^{m+\bar{\jmath}_{1}+\ldots+\bar{\jmath}_{m}} \in \mathrm{U}(\mathfrak{g})
$$

under the tensor product of the evaluation representations

$$
\mathrm{U}(\mathfrak{g}) \rightarrow \operatorname{End}\left(\mathbb{C}^{N \mid N}\right): F_{i j}^{(s)} \mapsto E_{i j} z^{s}+E_{-i,-j}(-z)^{s}, \quad s \geqslant 0
$$

at the points $z=z_{1}, \ldots, z_{n} \in \mathbb{C}$; see the definition $(2.15)$ of the element $F_{i j}^{(s)} \in \mathfrak{g}$, and the formula $(2.21)$ for the representation $\mathrm{Y}\left(\mathfrak{q}_{N}\right) \rightarrow \operatorname{End}\left(\mathbb{C}^{N \mid N}\right)$ corresponding to $z \in \mathbb{C}$. Due to Proposition 2.1 it now suffices to show that when $z_{1}, \ldots, z_{n} \in \mathbb{C}$ and the positive integer $n$ vary, the kernels of the tensor products of the evaluation representations of the algebra $\mathrm{U}(\mathfrak{g})$ at $z=z_{1}, \ldots, z_{n} \in \mathbb{C}$ have zero intersection. This will also imply that the homomorphism (2.16) is injective.

The algebra $U(\mathfrak{g})$ is a subalgebra in the universal enveloping algebra of the Lie superalgebra $\mathfrak{g l}_{N \mid N}[u]$. We will show that the intersection of the kernels of all finite tensor products of evaluation representations $\mathrm{U}\left(\mathfrak{g l}_{N \mid N}[u]\right) \rightarrow \operatorname{End}\left(\mathbb{C}^{N \mid N}\right)$, is zero. Denote by $\varpi_{n}$ the supersymmetrisation map in the tensor product $\left(\mathfrak{g l}_{N \mid N}[u]\right)^{\otimes n}$ normalised so that $\varpi_{n}^{2}=\varpi_{n}$. We will identify the vector space $\left(\mathfrak{g l}_{N \mid N}[u]\right)^{\otimes n}$ with $\mathfrak{g l}_{N \mid N}^{\otimes n}\left[u_{1}, \ldots, u_{n}\right]$ where $u_{1}, \ldots, u_{n}$ are independent complex variables. 
The vector space $\mathfrak{g l}_{N \mid N}$ is identified with $\operatorname{End}\left(\mathbb{C}^{N \mid N}\right)$. Choose any linear basis $X_{1}, \ldots, X_{4 N^{2}}$ in $\mathfrak{g l}_{N \mid N}$ such that $X_{1}=E$ as in (1.5). The element $X_{1} \in \mathfrak{g l}_{N \mid N}$ is then identified with the operator $1 \in \operatorname{End}\left(\mathbb{C}^{N \mid N}\right)$. Take any finite non-zero linear combination of the elements

$$
\left(X_{a_{1}} u^{s_{1}}\right) \ldots\left(X_{a_{m}} u^{s_{m}}\right) \in \mathrm{U}\left(\mathfrak{g l}_{N \mid N}[u]\right)
$$

where the indices $s_{1}, \ldots, s_{m} \geqslant 0$ and the number $m \geqslant 0$ may vary. We assume that for every fixed $m$ the elements

$$
\varpi_{m}\left(X_{a_{1}} u^{s_{1}} \otimes \ldots \otimes X_{a_{m}} u^{s_{m}}\right) \in\left(\mathfrak{g l}_{N \mid N}[u]\right)^{\otimes m}=\operatorname{End}\left(\mathbb{C}^{N \mid N}\right)^{\otimes m}\left[u_{1}, \ldots, u_{m}\right]
$$

are linearly independent. Further, we will suppose that in every product (2.23) the indices $a_{1}, \ldots, a_{p}>1$ for certain $p \leqslant m$ while $a_{p+1}=\ldots=a_{m}=1$. We will also suppose that $s_{p+1}, \ldots, s_{q}>0$ for some $q \geqslant p$, while $s_{q+1}=\ldots=s_{m}=0$.

For any $n \geqslant p$ consider the tensor product $\nu$ of the evaluation representations of the algebra $\mathrm{U}\left(\mathfrak{g l}_{N \mid N}[u]\right)$ at $u_{1}, \ldots, u_{n} \in \mathbb{C}$. Let us denote by $\mathrm{P}$ the subspace in $\operatorname{End}\left(\mathbb{C}^{N \mid N}\right)^{\otimes n}$ spanned by the vectors $X_{b_{1}} \otimes \ldots \otimes X_{b_{n}}$ where either the number of indices $b_{r}>1$ is less than $p$, or $b_{r}=1$ for at least one $r \leqslant p$. The image of (2.23) under $\nu$ is a polynomial in $u_{1}, \ldots, u_{n}$ valued in $\operatorname{End}\left(\mathbb{C}^{N \mid N}\right)^{\otimes n}$, of the form

$$
p ! \varpi_{p}\left(X_{a_{1}} u^{s_{1}} \otimes \ldots \otimes X_{a_{p}} u^{s_{p}}\right) \otimes 1^{\otimes(n-p)} \cdot \prod_{p<r \leqslant q}\left(u_{1}^{s_{r}}+\ldots+u_{n}^{s_{r}}\right) \cdot n^{m-q}
$$

plus the terms valued in the subspace $\mathrm{P} \subset \operatorname{End}\left(\mathbb{C}^{N \mid N}\right)^{\otimes n}$. Here the tensor factor $\varpi_{p}\left(X_{a_{1}} u^{s_{1}} \otimes \ldots \otimes X_{a_{p}} u^{s_{p}}\right)$ is regarded as an element of End $\left(\mathbb{C}^{N \mid N}\right)^{\otimes p}\left[u_{1}, \ldots, u_{p}\right]$ by identifying this algebra with $\left(\mathfrak{g l}_{N \mid N}[u]\right)^{\otimes p}$.

The numbers $p$ for various products (2.23) from our linear combination may differ. Take those products (2.23) where the number $p$ is maximal. For any $n \geqslant p$ the images of the remaining products under $\nu$ are polynomials in $u_{1}, \ldots, u_{n}$ taking values in the subspace $\mathrm{P} \subset \operatorname{End}\left(\mathbb{C}^{N \mid N}\right)^{\otimes n}$. But a non-zero linear combination of the polynomials (2.24) with the maximal $p$, cannot vanish identically for all $n \geqslant p$ by Poincaré-Birkhoff-Witt theorem [MM, Theorem 5.15] for Lie superalgebras

In the course of the proof of Proposition 2.2 we established that the homomorphism (2.16) is injective. Together with Proposition 2.1, this yields the following result.

Theorem 2.3. $\mathbb{Z}_{2}$-graded algebras $\mathrm{U}(\mathfrak{g})$ and $\operatorname{gr}_{-} \mathrm{Y}\left(\mathfrak{q}_{N}\right)$ are isomorphic via (2.16).

Let us now return to the first $\mathbb{Z}$-filtration on the algebra $\mathrm{Y}\left(\mathfrak{q}_{N}\right)$. Let $t_{i j}^{(s)}$ be the element of the algebra gr $\mathrm{Y}\left(\mathfrak{q}_{N}\right)$ corresponding to the generator $T_{i j}^{(s)} \in \mathrm{Y}\left(\mathfrak{q}_{N}\right)$. The algebra gr $\mathrm{Y}\left(\mathfrak{q}_{N}\right)$ inherits $\mathbb{Z}_{2}$-gradation from $\mathrm{Y}\left(\mathfrak{q}_{N}\right)$ such that $\operatorname{deg} t_{i j}^{(s)}=\bar{\imath}+\bar{\jmath}$.

Corollary 2.4. The algebra $\mathrm{gr} \mathrm{Y}\left(\mathfrak{q}_{N}\right)$ is supercommutative with free generators $t_{i j}^{(s)}$ and $t_{i,-j}^{(s)}$ where $s=1,2, \ldots$ and $i, j=1, \ldots, N$.

Proof. The $\mathbb{Z}$-graded algebra gr $\mathrm{Y}\left(\mathfrak{q}_{N}\right)$ is supercommutative due to the relations (2.2). Moreover, by (2.3) for any $s \geqslant 1$ we have the relation $t_{-i,-j}^{(s)}=(-1)^{s} t_{i j}^{(s)}$. The supercommuting generators $t_{i j}^{(s)}$ with $i>0$ are free due to Theorem 2.3 
To finish this section let us show that the Hopf superalgebra $\mathrm{Y}\left(\mathfrak{q}_{N}\right)$ provides a quantisation of the co-Poisson Hopf superalgebra $U(\mathfrak{g})$ in the sense of [D1]. Let $h$ be a formal parameter. Take the tensor product $\mathbb{C}[[h]] \otimes \mathrm{Y}\left(\mathfrak{q}_{N}\right)$ where $h$ has $\mathbb{Z}_{2}$-degree zero. Denote by $\mathrm{Y}\left(\mathfrak{q}_{N}, h\right)$ the unital subalgebra in this tensor product, generated by all the elements $H_{i j}^{(s)}=T_{i j}^{(s)} h^{s-1}$ with $s \geqslant 1$. Due to Theorem 2.3 an isomorphism of $\mathbb{Z}_{2}$-graded algebras $\mathrm{Y}\left(\mathfrak{q}_{N}, h\right) / h \mathrm{Y}\left(\mathfrak{q}_{N}, h\right) \rightarrow \mathrm{U}(\mathfrak{g})$ can be defined by

$$
H_{i j}^{(s)}+h \mathrm{Y}\left(\mathfrak{q}_{N}, h\right) \mapsto-F_{j i}^{(s-1)} \cdot(-1)^{\bar{\jmath}},
$$

see (2.15). Let us extend the comultuplication $\Delta$ to $\mathrm{Y}\left(\mathfrak{q}_{N}, h\right)$ by $\mathbb{C}[[h]]$-linearity. The definition (2.19) implies that the assignment (2.25) defines an isomorphism of Hopf superalgebras. Let $\psi: \mathrm{Y}\left(\mathfrak{q}_{N}, h\right) \rightarrow \mathrm{U}(\mathfrak{g})$ be the composition of the projection $\mathrm{Y}\left(\mathfrak{q}_{N}, h\right) \rightarrow \mathrm{Y}\left(\mathfrak{q}_{N}, h\right) / h \mathrm{Y}\left(\mathfrak{q}_{N}, h\right)$ with the isomorphism $(2.25)$.

Now let us consider the co-supercommutator $\varphi: \mathfrak{g} \rightarrow \mathfrak{g}^{\otimes 2} \subset \mathfrak{g l}_{N \mid N}[u] \otimes \mathfrak{g l}_{N \mid N}[v]$ determined by (1.3), where according to (1.7) we put

$$
r(u, v)=\sum_{i j} E_{i j} \otimes E_{j i} \cdot \frac{(-1)^{\bar{\jmath}}}{u-v}+\sum_{i j} E_{i j} \otimes E_{-j,-i} \cdot \frac{(-1)^{\bar{\jmath}}}{u+v} .
$$

Extend $\varphi$ to the co-Poisson bracket $\mathrm{U}(\mathfrak{g}) \rightarrow \mathrm{U}(\mathfrak{g})^{\otimes 2}$. Denote this extension by the same letter $\varphi$. Further, denote by $\Delta^{\circ}$ the composition of the comultiplication $\Delta$ on $\mathrm{Y}\left(\mathfrak{q}_{N}, h\right)$ with the involutive automorphism $\theta$ of the algebra $\mathrm{Y}\left(\mathfrak{q}_{N}, h\right)^{\otimes 2}$, defined in the beginning of Section 1. To show that $\mathrm{Y}\left(\mathfrak{q}_{N}, h\right)$ is a quantisation of the co-Poisson Hopf superalgebra $\mathrm{U}(\mathfrak{g})$ it remains to prove the following proposition.

Proposition 2.4. For any element $X \in \mathrm{Y}\left(\mathfrak{q}_{N}, h\right)$ we have the equality

$$
(\psi \otimes \psi)\left(\left(\Delta(X)-\Delta^{\circ}(X)\right) / h\right)=\varphi(\psi(X)) \text {. }
$$

Proof. If suffices to verify the equality (2.27) for the generators $H_{i j}^{(s)}$ of the algebra $\mathrm{Y}\left(\mathfrak{q}_{N}, h\right)$. By the definitions $(2.25)$ and $(1.3),(2.26)$ for $s \geqslant 1$ we have in $\mathrm{U}(\mathfrak{g})^{\otimes 2}$

$$
\begin{gathered}
\varphi\left(\psi\left(H_{i j}^{(s)}\right)\right)=-\varphi\left(F_{j i}^{(s-1)}\right) \cdot(-1)^{\bar{\jmath}}= \\
\sum_{1 \leqslant r \leqslant s-1}\left(F_{k i}^{(r-1)} \otimes F_{j k}^{(s-r-1)} \cdot(-1)^{(\bar{\imath}+\bar{k}+1)(\bar{\jmath}+\bar{k})}-F_{j k}^{(r-1)} \otimes F_{k i}^{(s-r-1)} \cdot(-1)^{\bar{\jmath}+\bar{k}}\right) .
\end{gathered}
$$

On the other hand, by the definition $(2.19)$ for any $s \geqslant 1$ we have in $\mathrm{Y}\left(\mathfrak{q}_{N}, h\right)^{\otimes 2}$

$$
\begin{aligned}
\Delta\left(H_{i j}^{(s)}\right) & =H_{i j}^{(s)} \otimes 1+1 \otimes H_{i j}^{(s)}+\sum_{1 \leqslant r \leqslant s-1} h \cdot H_{i k}^{(r)} \otimes H_{k j}^{(s-r)} \cdot(-1)^{(\bar{\imath}+\bar{k})(\bar{\jmath}+\bar{k})}, \\
\Delta^{\circ}\left(H_{i j}^{(s)}\right) & =H_{i j}^{(s)} \otimes 1+1 \otimes H_{i j}^{(s)}+\sum_{1 \leqslant r \leqslant s-1} h \cdot H_{k j}^{(r)} \otimes H_{i k}^{(s-r)} .
\end{aligned}
$$

Thus using again the definition (2.25) we get the equality $(2.27)$ for $X=H_{i j}^{(s)}$

\section{Centre of the Yangian}

In this section we will give a description of the centre of the $\mathbb{Z}_{2}$-graded algebra $\mathrm{Y}\left(\mathfrak{q}_{N}\right)$. By definition an element of $\mathrm{Y}\left(\mathfrak{q}_{N}\right)$ is central if it supercommutes with any element of $\mathrm{Y}\left(\mathfrak{q}_{N}\right)$. However, we will see that the centre of $\mathrm{Y}\left(\mathfrak{q}_{N}\right)$ consists of even elements only. We will use some arguments from [MNO, Proposition 2.12]. 
Let $\tau$ be the antiautomorphism of the $\mathbb{Z}_{2}$-graded algebra $\operatorname{End}\left(\mathbb{C}^{N \mid N}\right)$ defined by the assignment

$$
E_{i j} \mapsto E_{j i} \cdot(-1)^{\bar{\imath}(\bar{\jmath}+1)}
$$

for any $i$ and $j$. Introduce the element of the algebra $\operatorname{End}\left(\mathbb{C}^{N \mid N}\right)^{\otimes 2}$

$$
Q=\operatorname{id} \otimes \tau(P)=\sum_{i, j} E_{i j} \otimes E_{i j} \cdot(-1)^{\bar{\imath} \bar{\jmath}} .
$$

Denote

$$
\bar{T}(u)=\tau \otimes \operatorname{id}(\widetilde{T}(u)) \in \operatorname{End}\left(\mathbb{C}^{N \mid N}\right) \otimes \mathrm{Y}\left(\mathfrak{q}_{N}\right)\left[\left[u^{-1}\right]\right] .
$$

The following construction of central elements in $Y\left(\mathfrak{q}_{N}\right)$ goes back to [N1, Section 1]. Proposition 3.1. For a certain element $Z(u) \in \mathrm{Y}\left(\mathfrak{q}_{N}\right)\left[\left[u^{-1}\right]\right]$ we have the equality

$$
(Q \otimes 1) \cdot T_{1}(u) \bar{T}_{2}(u)=Q \otimes Z(u)
$$

in the algebra $\operatorname{End}\left(\mathbb{C}^{N \mid N}\right)^{\otimes 2} \otimes \mathrm{Y}\left(\mathfrak{q}_{N}\right)\left[\left[u^{-1}\right]\right]$. The coefficients of the series $Z(u)$ are of $\mathbb{Z}_{2}$-degree zero and belong to the centre of the algebra $\mathrm{Y}\left(\mathfrak{q}_{N}\right)$.

Proof. Introduce the rational function $\bar{R}(u, v)=\mathrm{id} \otimes \tau(R(u, v))$ valued in the algebra $\operatorname{End}\left(\mathbb{C}^{N \mid N}\right)^{\otimes 2}$. One can directly verify the identity

$$
\bar{R}(u, v) \bar{R}(-u,-v)=1 .
$$

By making use of this identity we derive from (2.7) the relation

$$
(\bar{R}(-u,-v) \otimes 1) \cdot T_{1}(u) \bar{T}_{2}(v)=\bar{T}_{2}(v) T_{1}(u) \cdot(\bar{R}(-u,-v) \otimes 1) .
$$

Let us multiply each side of this relation by $u-v$ and then put $u=v$. We obtain

$$
(Q \otimes 1) \cdot T_{1}(u) \bar{T}_{2}(u)=\bar{T}_{2}(u) T_{1}(u) \cdot(Q \otimes 1) .
$$

Since the image of the endomorphism $Q \in \operatorname{End}\left(\mathbb{C}^{N \mid N}\right)^{\otimes 2}$ has dimension one, we get the first statement of Proposition 3.1. Since $Q$ has $\mathbb{Z}_{2}$-degree zero, the equality (3.1) shows that every coefficient of the series $Z(u)$ has $\mathbb{Z}_{2}$-degree zero in $\mathrm{Y}\left(\mathfrak{q}_{N}\right)$.

Let us now work with the algebra End $\left(\mathbb{C}^{N \mid N}\right)^{\otimes 3} \otimes \mathrm{Y}\left(\mathfrak{q}_{N}\right)\left[\left[u^{-1}, v^{-1}\right]\right]$. Using the relations $(2.7),(3.2)$ and the definition (3.1) we get the equalities

$$
\begin{array}{r}
\left(Q_{23} \bar{R}_{13}(-u,-v) R_{12}(u, v) \otimes 1\right) \cdot T_{1}(u) T_{2}(v) \bar{T}_{3}(v)= \\
\left(Q_{23} \otimes 1\right) \cdot T_{2}(v) \bar{T}_{3}(v) T_{1}(u) \cdot\left(\bar{R}_{13}(-u,-v) R_{12}(u, v) \otimes 1\right)= \\
\left(Q_{23} \otimes Z(v)\right) \cdot T_{1}(u) \cdot\left(\bar{R}_{13}(-u,-v) R_{12}(u, v) \otimes 1\right) .
\end{array}
$$

On the other hand, by $(2.14)$ we have the identity in $\operatorname{End}\left(\mathbb{C}^{N \mid N}\right)^{\otimes 3}(u, v)$

So

$$
R_{13}(-u,-v) P_{23} R_{12}(u, v)=P_{23} \cdot\left(1-\frac{1}{(u-v)^{2}}-\frac{1}{(u+v)^{2}}\right) .
$$

$$
Q_{23} \bar{R}_{13}(-u,-v) R_{12}(u, v)=Q_{23} \cdot\left(1-\frac{1}{(u-v)^{2}}-\frac{1}{(u+v)^{2}}\right) .
$$

Due to the latter identity we obtain from (3.3) the equality

$$
T_{1}(u) \cdot Q_{23} \otimes Z(v)=Q_{23} \otimes Z(v) \cdot T_{1}(u) .
$$

Hence every coefficient of the series $Z(v)$ commutes with any generator $T_{i j}^{(s)}$ of the algebra $\mathrm{Y}\left(\mathfrak{q}_{N}\right)$ 
Let us consider the square $S^{2}$ of the antipodal map. It is an automorphism of the $\mathbb{Z}_{2}$-graded algebra $\mathrm{Y}\left(\mathfrak{q}_{N}\right)$. Here is an alternative definition of the series $Z(u)$.

Proposition 3.2. We have $S^{2}\left(T_{i j}(u)\right)=T_{i j}(u) \cdot Z^{-1}(u)$ for all indices $i$ and $j$.

Proof. Definition (3.1) is equvalent to the collection of relations in $\mathrm{Y}\left(\mathfrak{q}_{N}\right)\left[\left[u^{-1}\right]\right]$

$$
\sum_{i} T_{i j}(u) \widetilde{T}_{k i}(u)=Z(u) \delta_{j k} .
$$

On the other hand, by the definition of the anipodal map $S$ we have the relations

$$
\sum_{i} T_{k i}(u) S\left(T_{i j}(u)\right) \cdot(-1)^{(\bar{\imath}+\bar{k})(\bar{\imath}+\bar{\jmath})}=\delta_{j k} .
$$

By applying the antiautomorphism $S$ to each side of the latter equality we get

$$
\sum_{i} S^{2}\left(T_{i j}(u)\right) \widetilde{T}_{k i}(u)=\delta_{j k}
$$

By comparing the last equality with (3.4) we prove Proposition 3.2

Corollary 3.3. We have the equalities of formal series in $u^{-1}$

$$
\Delta(Z(u))=Z(u) \otimes Z(u), \quad \varepsilon(Z(u))=1, \quad S(Z(u))=Z^{-1}(u) .
$$

Proof. Let $\theta$ be the involutive automorphism of the algebra $\mathrm{Y}\left(\mathfrak{q}_{N}\right) \otimes \mathrm{Y}\left(\mathfrak{q}_{N}\right)$ as defined in the beginning of Section 1. Since $\Delta \circ S=\theta \circ(S \otimes S) \circ \Delta$ we get

$$
\Delta\left(\widetilde{T}_{i j}(u)\right)=\sum_{k} \widetilde{T}_{k j}(u) \otimes \widetilde{T}_{i k}(u)
$$

from the definition (2.19). Now by using (2.19) again we obtain the first equality in Corollary 3.3 from (3.4). The second equality follows directly from (3.4). To obtain the third equality in Corollary 3.3 apply the the antiautomorphism $S$ to each side of (3.4) and then use (3.5) along with Proposition 3.2

Observe that due to the relations $(2.3)$ we have $Z(-u)=Z(u)$. Thus

$$
Z(u)=1+Z^{(2)} u^{-2}+Z^{(4)} u^{-4}+\ldots
$$

for certain central elements $Z^{(2)}, Z^{(4)}, \ldots \in \mathrm{Y}\left(\mathfrak{q}_{N}\right)$. We have the following theorem. Theorem 3.4. Elements $Z^{(2)}, Z^{(4)}, \ldots$ are free generators of the centre of $\mathrm{Y}\left(\mathfrak{q}_{N}\right)$. We will present the main steps of the proof as separate propositions. We will make use of the second ascending filtration on the algebra $\mathrm{Y}\left(\mathfrak{q}_{N}\right)$. Take the element $G_{i j}^{(s)}$ of the $\mathbb{Z}$-graded algebra gr_Y $\left(\mathfrak{q}_{N}\right)$ corresponding to generator $T_{i j}^{(s)} \in \mathrm{Y}\left(\mathfrak{q}_{N}\right)$. Denote

$$
G^{(s)}=\sum_{i} G_{i i}^{(s)} \cdot(-1)^{\bar{\imath}} .
$$

Note that by the relation (2.3) here $G^{(s)}=0$ if the number $s$ is even. Theorem 2.3 provides an isomorphism between gr_$_{-} \mathrm{Y}\left(\mathfrak{q}_{N}\right)$ and the enveloping algebra $\mathrm{U}(\mathfrak{g})$ of the Lie superalgebra (1.6). In particular, the elements $G^{(1)}, G^{(3)}, \ldots \in \operatorname{gr}_{-} \mathrm{Y}\left(\mathfrak{q}_{N}\right)$ are algebraically independent. 
Proposition 3.5. For any index $s=2,4, \ldots$ the element of the algebra gr_$_{-} \mathrm{Y}\left(\mathfrak{q}_{N}\right)$ corresponding to $Z^{(s)} \in \mathrm{Y}\left(\mathfrak{q}_{N}\right)$ is $(s-1) \cdot G^{(s-1)}$.

Proof. Amongst other relations the collection (3.2) contains the equality

$$
\left[T_{i j}(u), \widetilde{T}_{j i}(v)\right]=\frac{(-1)^{\bar{\imath}}}{u-v} \sum_{k}\left(T_{k j}(u) \widetilde{T}_{j k}(v)-\widetilde{T}_{k i}(v) T_{i k}(u)\right)
$$

for any indices $i$ and $j$. The square brackets here stand for the supercommutator. By performing summation in this equality over the index $i$ we get

$$
\sum_{i} T_{i j}(u) \widetilde{T}_{j i}(v)=1-\sum_{i, k} \widetilde{T}_{k i}(v) T_{i k}(u) \cdot \frac{(-1)^{\bar{\imath}}}{u-v}
$$

By setting $u$ equal to $v$ in the latter equality we obtain due to (3.4) that

$$
Z(v)=1-\sum_{i, k} \widetilde{T}_{k i}(v) \dot{T}_{i k}(v) \cdot(-1)^{\bar{\imath}}
$$

where

$$
\dot{T}_{i k}(v)=-T_{i k}^{(1)} v^{-2}-2 T_{i k}^{(2)} v^{-3}-\ldots
$$

is the first derivative of the formal series $T_{i k}(v)$ with respect to the parameter $v$. By the definition of the second filtration on $Y\left(\mathfrak{q}_{N}\right)$ the element of the $\mathbb{Z}$-graded algebra gr_Y $\left(\mathfrak{q}_{N}\right)$ corresponding to the coefficient at $v^{-s}$ in the expansion of the right hand side of $(3.6)$ is $(s-1) \cdot G^{(s-1)}$ whenever $s \geqslant 1$

To prove Theorem 3.4 it suffices to show that the elements $G^{(1)}, G^{(3)}, \ldots$ generate the centre of gr_ $_{-} \mathrm{Y}\left(\mathfrak{q}_{N}\right)$. By Theorem 2.3 this means that for the element $E \in \mathfrak{q}_{N}$ defined by (1.5), the elements $E, E u^{2}, E u^{4}, \ldots \in \mathfrak{g}$ generate the centre of the $\mathbb{Z}_{2}$-graded algebra $\mathrm{U}(\mathfrak{g})$.

To prove the latter statement we will consider the following general situation. Let $\mathfrak{b}$ be an arbitrary finite-dimensional Lie superalgebra. Let $\omega$ be any involutive automorphism of $\mathfrak{b}$. Consider the corresponding twisted polynomial current Lie superalgebra

$$
\mathfrak{h}=\{X(u) \in \mathfrak{b}[u]: \omega(X(u))=X(-u)\} .
$$

Proposition 3.6. Suppose that the centre of the Lie superalgebra $\mathfrak{b}$ is trivial. Then the centre of the universal enveloping algebra $\mathrm{U}(\mathfrak{h})$ is also trivial.

Proof. We will prove that the adjoint action of $\mathfrak{h}$ in the supercommutative algebra $\mathrm{S}(\mathfrak{h})$ has only trivial invariant elements. Choose a homogeneous basis $X_{1}, \ldots, X_{n}$ in $\mathfrak{b}$ and let

$$
\left[X_{p}, X_{q}\right]=\sum_{r} c_{p q r} X_{r}
$$

where $c_{p q r} \in \mathbb{C}$ is a structure constant of $\mathfrak{b}$. We put $\bar{r}=0$ if the element $X_{r} \in \mathfrak{b}$ is even and $\bar{r}=1$ if this element is odd. Assume that for some $h \leqslant n$ we have $\omega\left(X_{r}\right)=X_{r}$ when $1 \leqslant r \leqslant h$ and $\omega\left(X_{r}\right)=-X_{r}$ when $h<r \leqslant n$.

The elements $X_{r} t^{s}$ where $1 \leqslant r \leqslant h$ when $s=0,2, \ldots$ and $h<r \leqslant n$ when $s=1,3, \ldots$ form a basis in the Lie superalgebra $\mathfrak{h}$. Let us order the set of the pairs $(s, r)$ here lexicographically:

$$
(0,1) \prec \ldots \prec(0, h) \prec(1, h+1) \prec \ldots \prec(1, n) \prec \ldots
$$


A basis in the supercommutative algebra $S(\mathfrak{h})$ is then formed by all finite ordered products of the elements $\left(X_{r} u^{s}\right)^{d}$ over the set of pairs $(s, r)$ where $d=0,1,2, \ldots$ when $\bar{r}=0$ but $d=0,1$ when $\bar{r}=1$.

Let us now fix any $\mathfrak{h}$-invariant element $Y \in \mathrm{S}(\mathfrak{h})$. Let $m$ be the maximal integer such that $X_{r} u^{m}$ occurs in $Y$ for some index $r$. Suppose that $m$ is even. Then the element $Y$ is a finite sum

$$
Y=\sum_{d_{1} \ldots d_{h}} Y_{d_{1} \ldots d_{h}} \cdot\left(X_{1} u^{m}\right)^{d_{1}} \ldots\left(X_{h} u^{m}\right)^{d_{h}}
$$

where any factor $Y_{d_{1} \ldots d_{h}} \in \mathrm{S}(\mathfrak{h})$ depends only on elements $X_{r} u^{s} \in \mathfrak{h}$ with $s<m$. This factor is zero if $d_{p}>1$ for some index $p \leqslant h$ with $\bar{p}=1$. By our assumption

$$
\operatorname{ad}\left(X_{q} u\right) \cdot Y=0 ; \quad q=h+1, \ldots, n .
$$

The minimal component of the left hand side of (3.7) that depends on elements $X_{r} u^{m+1} \in \mathfrak{h}$ is the sum over $d_{1}, \ldots, d_{h}$ of the products in $\mathrm{S}(\mathfrak{h})$

$$
Y_{d_{1} \ldots d_{h}} \sum_{p \leqslant h}\left(X_{1} u^{m}\right)^{d_{1}} \ldots\left(X_{p} u^{m}\right)^{d_{p}-1} \ldots\left(X_{h} u^{m}\right)^{d_{h}} \sum_{h<r \leqslant n} d_{p} c_{p q r}\left(X_{r} u^{m+1}\right) \cdot(-1)^{f_{p}}
$$

where

$$
f_{p}=\sum_{p<s \leqslant h} \bar{s} d_{s}(\bar{q}+\bar{r})=\sum_{p<s \leqslant h} \bar{s} d_{s} \bar{p}
$$

if $c_{p q r} \neq 0$. That component must be equal to zero. So for all $q, r=h+1, \ldots, n$

$$
\sum_{d_{1} \ldots d_{h}} Y_{d_{1} \ldots d_{h}} \cdot \sum_{p \leqslant h}\left(X_{1} u^{m}\right)^{d_{1}} \ldots\left(X_{p} u^{m}\right)^{d_{p}-1} \ldots\left(X_{h} u^{m}\right)^{d_{h}} \cdot d_{p} c_{p q r}(-1)^{f_{p}}=0 .
$$

Thus for any sequence $d_{1}, \ldots, d_{h}$ of non-negative integers such that $d_{p} \leqslant 1$ if $\bar{p}=1$

$$
\sum_{p \leqslant h} Y_{d_{1} \ldots d_{p}+1 \ldots d_{h}}\left(d_{p}+1\right) c_{p q r} \cdot(-1)^{f_{p}}=0 ; \quad q, r=h+1, \ldots, n
$$

By our assumption we have along with (3.7) the collection of equalities in $\mathrm{S}(\mathfrak{h})$

$$
\operatorname{ad}\left(X_{q} u^{2}\right) \cdot Y=0 ; \quad q=1, \ldots, h
$$

By considering the minimal component of the left hand side of (3.9) that depends on elements $X_{r} u^{m+2} \in \mathfrak{h}$ we get along with (3.8) the equalities

$$
\sum_{p \leqslant h} Y_{d_{1} \ldots d_{p}+1 \ldots d_{h}}\left(d_{p}+1\right) c_{p q r} \cdot(-1)^{f_{p}}=0 ; \quad q, r=1, \ldots, h
$$

Let us now make use of the assumption that the centre of the Lie superalgebra $\mathfrak{h}$ is trivial. It implies that the system of linear equations on the variables $z_{1}, \ldots, z_{h}$

$$
\sum_{p \leqslant h}\left[z_{p}\left(d_{p}+1\right) X_{p} \cdot(-1)^{f_{p}}, X_{q}\right]=0 ; \quad q=1, \ldots, n
$$


has only the trivial solution. Rewrite the latter system as

$$
\sum_{p \leqslant h} z_{p}\left(d_{p}+1\right) c_{p q r} \cdot(-1)^{f_{p}}=0 ; \quad q, r=1, \ldots, n
$$

and compare the result with $(3.8),(3.10)$. We obtain that $Y_{d_{1} \ldots d_{p}+1 \ldots d_{h}}=0$ for any index $p=1, \ldots, h$. The sequence $d_{1}, \ldots, d_{h}$ here can be chosen arbitrarily. So we get $Y=Y_{0}, \ldots, 0$. The case when $m$ is odd can be treated similarly

By applying Proposition 3.6 to the quotient Lie superalgebra $\mathfrak{b}=\mathfrak{g l}_{N \mid N} / \mathbb{C} \cdot E$ we complete the proof of Theorem 3.4. Before closing this section let us consider the images in $\mathrm{U}\left(\mathfrak{q}_{N}\right)$ of the elements $Z^{(2)}, Z^{(4)}, \ldots \in \mathrm{Y}\left(\mathfrak{q}_{N}\right)$ with respect to the evaluation homomorphism $\pi_{N}$. By the definition (2.12) we obtain from (3.6) that

and

$$
\pi_{N}: Z^{(2)} \mapsto-\sum_{k} F_{k k}=-2 E
$$

$$
\pi_{N}: Z^{(s+2)} \mapsto-\sum_{k_{1}, \ldots, k_{s+1}} F_{k_{2} k_{1}} \ldots F_{k_{s+1} k_{s}} F_{k_{1} k_{s+1}} \cdot(-1)^{\bar{k}_{1}+\ldots+\bar{k}_{s}}
$$

for each $s=2,4, \ldots$ where the indices $k, k_{1}, \ldots, k_{s+1}$ run through $\pm 1, \ldots, \pm N$. So the elements $\pi_{N}\left(Z^{(2)}\right), \pi_{N}\left(Z^{(4)}\right), \ldots$ generate the centre of the algebra $\mathrm{U}\left(\mathfrak{q}_{N}\right)$ by [S1, Theorem 1]. In particular, we have the following corollary to Theorem 3.4.

Corollary 3.7. The image of the centre of $\mathrm{Y}\left(\mathfrak{q}_{N}\right)$ with respect to the evaluation homomorphism $\pi_{N}$ coincides with centre of the $\mathbb{Z}_{2}$-graded algebra $\mathrm{U}\left(\mathfrak{q}_{N}\right)$.

Different construction of a distinguished linear basis in the centre of the $\mathbb{Z}_{2}$-graded algebra $\mathrm{U}\left(\mathfrak{q}_{N}\right)$ was given in [N3, Section 4]. In particular, that construction yields a $\mathfrak{q}_{N}$-analogue of the classical Capelli identity [C] for the enveloping algebra $\mathrm{U}\left(\mathfrak{g l}_{N}\right)$. Results of the next section are underlying for that construction, cf. [N4, Section 3].

\section{Double of the Yangian}

The general notion of a quantum double was introduced in [D3, Section 13]. Here we consider the quantum double of the Yangian $\mathrm{Y}\left(\mathfrak{q}_{N}\right)$; cf. [S] and [BL, Section 3.3]. We employ it to define the universal $R$-matrix for the Hopf superalgebra $\mathrm{Y}\left(\mathfrak{q}_{N}\right)$.

Firstly consider a complex associative unital $\mathbb{Z}_{2}$-graded algebra $\mathrm{Y}^{*}\left(\mathfrak{q}_{N}\right)$ with the countable set of generators $T_{i j}^{(-s)}$ where $s=1,2, \ldots$ and $i, j= \pm 1, \ldots, \pm N$.

The $\mathbb{Z}_{2}$-gradation on the algebra $\mathrm{Y}^{*}\left(\mathfrak{q}_{N}\right)$ is determined by setting $\operatorname{deg} T_{i j}^{(-s)}=\bar{\imath}+\bar{\jmath}$ for each $s \geqslant 1$. To write down defining relations for these generators we put

$$
T_{i j}^{*}(v)=\delta_{i j} \cdot 1+T_{i j}^{(-1)}+T_{i j}^{(-2)} v+T_{i j}^{(-3)} v^{2}+\ldots \in \mathrm{Y}^{*}\left(\mathfrak{q}_{N}\right)[[v]] .
$$

Let us now combine all the series (4.1) into the single element

$$
T^{*}(v)=\sum_{i, j} T_{i j}^{*}(v) \otimes E_{i j} \in \mathrm{Y}^{*}\left(\mathfrak{q}_{N}\right) \otimes \operatorname{End}\left(\mathbb{C}^{N \mid N}\right)[[v]]
$$

Further, for any positive integer $n$ and each $s=1, \ldots, n$ we will denote

$$
T_{s}^{*}(v)=\mathrm{id} \otimes \iota_{s}\left(T^{*}(v)\right) \in \mathrm{Y}^{*}\left(\mathfrak{q}_{N}\right) \otimes \operatorname{End}\left(\mathbb{C}^{N \mid N}\right)^{\otimes n}[[v]]
$$


Then the defining relations in $\mathrm{Y}^{*}\left(\mathfrak{q}_{N}\right)$ can be written as

$$
\begin{aligned}
T_{1}^{*}(u) T_{2}^{*}(v) \cdot(1 \otimes R(u, v)) & =(1 \otimes R(u, v)) \cdot T_{2}^{*}(v) T_{1}^{*}(u), \\
\operatorname{id} \otimes \eta\left(T^{*}(v)\right) & =T^{*}(-v) .
\end{aligned}
$$

After multiplying each side of (4.3) by $u^{2}-v^{2}$ it becomes a relation in the algebra

$$
\mathrm{Y}^{*}\left(\mathfrak{q}_{N}\right) \otimes \operatorname{End}\left(\mathbb{C}^{N \mid N}\right)^{\otimes 2}[[u, v]]
$$

It is equivalent to the collection of relations in the algebra $\mathrm{Y}^{*}\left(\mathfrak{q}_{N}\right)[[u, v]]$

$$
\begin{gathered}
\left(u^{2}-v^{2}\right) \cdot\left[T_{i j}^{*}(u), T_{k l}^{*}(v)\right] \cdot(-1)^{\bar{\imath} \bar{\jmath}+\bar{\imath} \bar{l}+\bar{\jmath} \bar{l}}= \\
(u+v) \cdot\left(T_{i l}^{*}(u) T_{k j}^{*}(v)-T_{i l}^{*}(v) T_{k j}^{*}(u)\right)+ \\
(u-v) \cdot\left(T_{i,-l}^{*}(u) T_{k,-j}^{*}(v)-T_{-i, l}^{*}(v) T_{-k, j}^{*}(u)\right) \cdot(-1)^{\bar{\imath}+\bar{\jmath}}
\end{gathered}
$$

for all possible indices $i, j$ and $k, l$. Then (4.4) is equivalent to the collection of

$$
T_{i j}^{*}(-v)=T_{-i,-j}^{*}(v)
$$

There is a natural structure of $\mathbb{Z}_{2}$-graded bialgebra on $\mathrm{Y}^{*}\left(\mathfrak{q}_{N}\right)$. Due to (4.3) and (4.4) we can define a comultiplication $\Delta: \mathrm{Y}^{*}\left(\mathfrak{q}_{N}\right) \rightarrow \mathrm{Y}^{*}\left(\mathfrak{q}_{N}\right) \otimes \mathrm{Y}^{*}\left(\mathfrak{q}_{N}\right)$ by

$$
T_{i j}^{*}(v) \mapsto \sum_{k} T_{i k}^{*}(v) \otimes T_{k j}^{*}(v) \cdot(-1)^{(\bar{\imath}+\bar{k})(\bar{\jmath}+\bar{k})}
$$

similarly to (2.19). But here the tensor product is taken over the subalgebra $\mathbb{C}[[v]]$. The counit $\varepsilon: \mathrm{Y}^{*}\left(\mathfrak{q}_{N}\right) \rightarrow \mathbb{C}$ is determined so that $\varepsilon: T_{i j}^{(-s)} \mapsto 0$ for $s \geqslant 1$. Note that $\mathrm{Y}^{*}\left(\mathfrak{q}_{N}\right)$ is a bi-superalgebra but not a Hopf superalgebra. The antipode is defined for a completion $\mathrm{Y}^{\prime}\left(\mathfrak{q}_{N}\right)$ of $\mathrm{Y}^{*}\left(\mathfrak{q}_{N}\right)$ such that $T^{*}(0) \in \mathrm{Y}^{\prime}\left(\mathfrak{q}_{N}\right) \otimes \operatorname{End}\left(\mathbb{C}^{N \mid N}\right)$ is invertible. We will construct such a completion later in this section.

There is a canonical bilinear pairing $\langle\rangle:, \mathrm{Y}\left(\mathfrak{q}_{N}\right) \times \mathrm{Y}^{*}\left(\mathfrak{q}_{N}\right) \rightarrow \mathbb{C}$. We shall describe the corresponding linear map $\beta: \mathrm{Y}\left(\mathfrak{q}_{N}\right) \otimes \mathrm{Y}^{*}\left(\mathfrak{q}_{N}\right) \rightarrow \mathbb{C}$. The latter map will be defined following [RTF, Section 2] so that for all numbers $m, n=0,1,2, \ldots$

$$
\begin{array}{r}
\operatorname{End}\left(\mathbb{C}^{N \mid N}\right)^{\otimes m} \otimes \mathrm{Y}\left(\mathfrak{q}_{N}\right) \otimes \mathrm{Y}^{*}\left(\mathfrak{q}_{N}\right) \otimes \operatorname{End}\left(\mathbb{C}^{N \mid N}\right)^{\otimes n} \rightarrow \operatorname{End}\left(\mathbb{C}^{N \mid N}\right)^{\otimes(m+n)}: \\
T_{1}\left(u_{1}\right) \ldots T_{m}\left(u_{m}\right) \otimes T_{1}^{*}\left(v_{1}\right) \ldots T_{n}^{*}\left(v_{n}\right) \mapsto \prod_{1 \leqslant k \leqslant m}^{\rightarrow}\left(\prod_{1 \leqslant l \leqslant n}^{\rightarrow} R_{k, m+l}\left(u_{k}, v_{l}\right)\right)
\end{array}
$$

under the map id $\otimes \beta \otimes \mathrm{id}$. Here $u_{1}, \ldots, u_{m}, v_{1}, \ldots, v_{n}$ are independent variables and the product of the rational functions $R_{k, m+l}\left(u_{k}, v_{l}\right)$ should be expaned as a formal power series in $u_{1}^{-1}, \ldots, u_{m}^{-1}, v_{1}, \ldots, v_{n}$. In particular, when $m=n=0$ we get the equality $\langle 1,1\rangle=1$. Due to the relations $(2.7),(2.9)$ and $(4.3),(4.4)$ the consistency of this definition follows from $(2.10),(2.11)$ and (2.8). The following lemma describes a basic property of the pairing $\langle$,$\rangle .$ 
Lemma 4.1. Let $s_{1}, \ldots, s_{m}$ and $r_{1}, \ldots, r_{n}$ be any numbers from $\{1,2, \ldots\}$. Then

$$
\left\langle T_{i_{1} j_{1}}^{\left(s_{1}\right)} \ldots T_{i_{m} j_{m}}^{\left(s_{m}\right)}, T_{i_{m+1} j_{m+1}}^{\left(-r_{1}\right)} \ldots T_{i_{m+n} j_{m+n}}^{\left(-r_{n}\right)}\right\rangle \neq 0 \Rightarrow s_{1}+\ldots+s_{m} \geqslant r_{1}+\ldots+r_{n}
$$

for all $m, n=0,1,2, \ldots$ and any choice of the indices $i_{1}, j_{1}, \ldots, i_{m+n}, j_{m+n}$.

Proof. First suppose that $r_{1}, \ldots, r_{n} \geqslant 2$. Then by our definition the value of the pairing in Lemma 4.1 is up to the factor \pm 1 the coefficient at

$$
E_{i_{1} j_{1}} \otimes \ldots \otimes E_{i_{m+n} j_{m+n}} \cdot u_{1}^{-s_{1}} \ldots u_{m}^{-s_{m}} \cdot v_{1}^{r_{1}-1} \ldots v_{n}^{r_{n}-1}
$$

in the expansion of the product in $\operatorname{End}\left(\mathbb{C}^{N \mid N}\right)^{\otimes(m+n)}\left[\left[u_{1}^{-1}, \ldots, u_{m}^{-1}, v_{1}, \ldots, v_{n}\right]\right]$

$$
\prod_{1 \leqslant k \leqslant m}^{\rightarrow}\left(\prod_{1 \leqslant l \leqslant n}^{\rightarrow}\left(1-\sum_{s \geqslant 1} \frac{v_{l}^{s-1}}{u_{k}^{s}} P_{k, m+l}\left(1+(-1)^{s} J_{k} J_{m+l}\right)\right)\right)
$$

where we have used (2.6). If here the coefficient at (4.9) is non-zero then evidently

$$
s_{1}+\ldots+s_{m} \geqslant r_{1}+\ldots+r_{n} .
$$

Now suppose that some of the numbers $r_{1}, \ldots, r_{n}$ are equal to 1 . Without loss of generality we will assume that $r_{1}, \ldots, r_{p} \geqslant 2$ and $r_{p+1}, \ldots, r_{n}=1$ for some $p<n$. Rewrite the product over the indices $k, l$ at the right-hand side of (4.8) as

$$
\prod_{1 \leqslant l \leqslant p}\left(\prod_{1 \leqslant k \leqslant m}^{\rightarrow} R_{k, m+l}\left(u_{k}, v_{l}\right)\right) \cdot \overrightarrow{\prod_{p<l \leqslant n}}\left(\prod_{1 \leqslant k \leqslant m}^{\rightarrow} R_{k, m+l}\left(u_{k}, v_{l}\right)\right)
$$

Now the value of the pairing in Lemma 4.1 is up to the factor \pm 1 the coefficient at (4.9) in the expansion of the product

$$
\begin{aligned}
& \prod_{1 \leqslant l \leqslant p}^{\rightarrow}\left(\prod_{1 \leqslant k \leqslant m}^{\rightarrow}\left(1-\sum_{s \geqslant 1} \frac{v_{l}^{s-1}}{u_{k}^{s}} P_{k, m+l}\left(1+(-1)^{s} J_{k} J_{m+l}\right)\right)\right) \times \\
& \prod_{p<l \leqslant n}^{\rightarrow}\left(\prod_{1 \leqslant k \leqslant m}^{\rightarrow}\left(1-\sum_{s \geqslant 1} \frac{v_{l}^{s-1}}{u_{k}^{s}} P_{k, m+l}\left(1+(-1)^{s} J_{k} J_{m+l}\right)\right)-1\right)
\end{aligned}
$$

If here that coefficient is non-zero then $s_{1}+\ldots+s_{m} \geqslant r_{1}+\ldots+r_{p}+n-p$

We will equip the algebra $\mathrm{Y}^{*}\left(\mathfrak{q}_{N}\right)$ with the descending $\mathbb{Z}$-filtration defined by assigning to the generator $T_{i j}^{(-s)}$ the degree $s$ for any $s \geqslant 1$. The corresponding $\mathbb{Z}$-graded algebra will be denoted by $\operatorname{gr} \mathrm{Y}^{*}\left(\mathfrak{q}_{N}\right)$. The formal completion of the algebra $\mathrm{Y}^{*}\left(\mathfrak{q}_{N}\right)$ with respect to this filtration will be denoted by $\mathrm{Y}^{\prime}\left(\mathfrak{q}_{N}\right)$. We will extend the comultiplication $\Delta$ on $\mathrm{Y}^{*}\left(\mathfrak{q}_{N}\right)$ to the algebra $\mathrm{Y}^{\prime}\left(\mathfrak{q}_{N}\right)$, and still denote this extension by $\Delta$. The image $\Delta\left(\mathrm{Y}^{\prime}\left(\mathfrak{q}_{N}\right)\right)$ lies in the formal completion of the algebra $\mathrm{Y}^{*}\left(\mathfrak{q}_{N}\right) \otimes \mathrm{Y}^{*}\left(\mathfrak{q}_{N}\right)$ with respect to the descending $\mathbb{Z}$-filtration, defined by assigning to the element $T_{i j}^{(-r)} \otimes T_{k l}^{(-s)}$ the degree $r+s$. Indeed, with respect to this filtration $\Delta\left(T_{i j}^{(-r)}\right)$ is a finite sum of elements of degree not less than $r$. 
Let $G_{i j}^{(-s)} \in \mathrm{Y}^{*}\left(\mathfrak{q}_{N}\right)$ be the element corresponding to the generator $T_{i j}^{(-s)}$ of the algebra $\mathrm{Y}^{*}\left(\mathfrak{q}_{N}\right)$. The algebra grY $\mathrm{Y}^{*}\left(\mathfrak{q}_{N}\right)$ inherits $\mathbb{Z}_{2}$-gradation from $\mathrm{Y}^{*}\left(\mathfrak{q}_{N}\right)$ such that for any $s \geqslant 1$ we have $\operatorname{deg} t_{i j}^{(-s)}=\bar{\imath}+\bar{\jmath}$. By the relations (4.6) we have

$$
G_{-i,-j}^{(-s)}=(-1)^{s+1} G_{i j}^{(-s)}, \quad s \geqslant 1 .
$$

Furthermore, we can define a bilinear pairing

$$
\langle,\rangle: \operatorname{grY}\left(\mathfrak{q}_{N}\right) \times \operatorname{gr} Y^{*}\left(\mathfrak{q}_{N}\right) \rightarrow \mathbb{C}
$$

by making

$$
\left\langle t_{i_{1} j_{1}}^{\left(s_{1}\right)} \ldots t_{i_{m} j_{m}}^{\left(s_{m}\right)}, G_{i_{m+1} j_{m+1}}^{\left(-r_{1}\right)} \ldots G_{i_{m+n} j_{m+n}}^{\left(-r_{n}\right)}\right\rangle
$$

equal to

$$
\left\langle T_{i_{1} j_{1}}^{\left(s_{1}\right)} \ldots T_{i_{m} j_{m}}^{\left(s_{m}\right)}, T_{i_{m+1} j_{m+1}}^{\left(-r_{1}\right)} \ldots T_{i_{m+n} j_{m+n}}^{\left(-r_{n}\right)}\right\rangle
$$

if $s_{1}+\ldots+s_{m}=r_{1}+\ldots+r_{n}$ and equal to zero otherwise. Here $m, n \geqslant 0$ and $s_{1}, \ldots, s_{m}, r_{1}, \ldots, r_{n} \geqslant 1$ while the indices $i_{1}, j_{1}, \ldots, i_{m+n}, j_{m+n}$ are arbitrary. This definition is correct due to Lemma 4.1. Now for each $s=0,1,2, \ldots$ denote by $\operatorname{gr}_{s} \mathrm{Y}\left(\mathfrak{q}_{N}\right)$ and $\operatorname{gr}_{s} \mathrm{Y}^{*}\left(\mathfrak{q}_{N}\right)$ the subspaces of degree $s$ in the $\mathbb{Z}$-graded algebras $\operatorname{grY}\left(\mathfrak{q}_{N}\right)$ and $\operatorname{gr} Y^{*}\left(\mathfrak{q}_{N}\right)$ respectively.

Lemma 4.2. Restriction of the pairing (4.12) to $\operatorname{gr}_{s} \mathrm{Y}\left(\mathfrak{q}_{N}\right) \times \operatorname{gr}_{s} \mathrm{Y}^{*}\left(\mathfrak{q}_{N}\right)$ is not degenerate for any $s \geqslant 0$.

Proof. Fix any integers $s_{1}, \ldots, s_{m}, r_{1}, \ldots, r_{n} \geqslant 1$ such that

$$
s_{1}+\ldots+s_{m}=s_{m+1}+\ldots+s_{n} .
$$

Without loss of generality we will assume that $s_{1} \geqslant \ldots \geqslant s_{m}$ and $s_{m+1} \geqslant \ldots \geqslant s_{n}$. Suppose that $r_{1}, \ldots, r_{p} \geqslant 2$ while $r_{p+1}, \ldots, r_{n}=1$ for some $p \geqslant 0$. Now we do not exclude the case $p=n$. Let us consider the coefficient at

$$
u_{1}^{-s_{1}} \ldots u_{m}^{-s_{m}} \cdot v_{1}^{r_{1}-1} \ldots v_{n}^{r_{n}-1}
$$

in the expansion of the product (4.10) as a series in $u_{1}^{-1}, \ldots, u_{m}^{-1}, v_{1}, \ldots, v_{n}$. By our assumptions this coefficient can be non-zero only if $m=n$ and $s_{k}=r_{k}$ for all indices $k=1, \ldots, m$. Suppose that this is the case. For $r=1,2, \ldots$ denote by $\mathcal{S}_{r}$ the segment of the sequence $1, \ldots, m$ consisting of all $k$ such that $s_{k}=r$. Then the coefficient at (4.13) in the expansion of (4.10) equals

$$
(-1)^{m} \cdot \prod_{r \geqslant 1}\left(\sum_{g} \prod_{l \in \mathcal{S}_{r}} P_{g(l), m+l}\left(1+(-1)^{r} J_{g(l)} J_{m+l}\right)\right)
$$

where the index $g$ runs through the set of all permutations of the sequence $\mathcal{S}_{r}$. Note that the factors in each of the above two products commute.

Choose any basis in the space $\operatorname{gr}_{s} \mathrm{Y}\left(\mathfrak{q}_{N}\right)$ consisting of monomials $t_{i_{1} j_{1}}^{\left(s_{1}\right)} \ldots t_{i_{m} j_{m}}^{\left(s_{m}\right)}$ such that

$$
s_{1} \geqslant \ldots \geqslant s_{m} \geqslant 1, \quad s_{1}+\ldots+s_{m}=s
$$


and

$$
i_{k} \in\{1, \ldots, N\}, \quad j_{k} \in\{ \pm 1, \ldots, \pm N\}
$$

for $k=1, \ldots, m$ while the number $m \geqslant 0$ can vary. The above argument using the expression (4.14) shows that for any two elements of this basis

$$
t_{i_{1} j_{1}}^{\left(s_{1}\right)} \ldots t_{i_{m} j_{m}}^{\left(s_{m}\right)} \quad \text { and } \quad t_{i_{m+1} j_{m+1}}^{\left(r_{1}\right)} \ldots t_{i_{m+n} j_{m+n}}^{\left(r_{n}\right)}
$$

the value

$$
\left\langle t_{i_{1} j_{1}}^{\left(s_{1}\right)} \ldots t_{i_{m} j_{m}}^{\left(s_{m}\right)}, G_{j_{m+1} i_{m+1}}^{\left(-r_{1}\right)} \ldots G_{j_{m+n} i_{m+n}}^{\left(-r_{n}\right)}\right\rangle
$$

is non-zero only if $m=n$ and for each index $k=1, \ldots, m$ we have the equalities

$$
i_{m+k}=i_{k}, \quad j_{m+k}=j_{k}, \quad r_{k}=s_{k}
$$

In the latter case that value up to the factor \pm 1 is the product $a ! b ! \ldots$ where $a, b, \ldots$ are multiplicities in the sequence of the triples $\left(i_{1}, j_{1}, s_{1}\right), \ldots,\left(i_{m}, j_{m}, s_{m}\right)$. But the products

$$
G_{j_{1} i_{1}}^{\left(-s_{1}\right)} \ldots G_{j_{m} i_{m}}^{\left(-s_{m}\right)} \in \operatorname{gr} \mathrm{Y}^{*}\left(\mathfrak{q}_{N}\right)
$$

corresponding to elements of our basis in $\operatorname{gr}_{s} \mathrm{Y}\left(\mathfrak{q}_{N}\right)$ span the space $\operatorname{gr}_{s} \mathrm{Y}^{*}\left(\mathfrak{q}_{N}\right)$

Take the subalgebra $\mathfrak{g}^{\prime}=u \cdot \mathfrak{g}$ in the Lie superalgebra $\mathfrak{g l}_{N \mid N}[u]$, see definition (1.6). Consider the corresponding universal enveloping algebra $U\left(\mathfrak{g}^{\prime}\right)$.

Corollary 4.3. The $\mathbb{Z}_{2}$-graded algebras $\operatorname{gr} \mathrm{Y}^{*}\left(\mathfrak{q}_{N}\right)$ and $\mathrm{U}\left(\mathfrak{g}^{\prime}\right)$ are isomorphic.

Proof. Consider the elements $F_{i j}^{(s)}$ of the universal enveloping algebra of $\mathfrak{g l}_{N \mid N}[u]$ with $s \geqslant 0$, defined by (2.15). Any relation between these elements follows from $(2.17),(2.18)$. On the other hand, the generators $G_{i j}^{(-s)}$ of the algebra $\operatorname{gr} \mathrm{Y}^{*}\left(\mathfrak{q}_{N}\right)$ with $s \geqslant 1$ satisfy (4.11). Due to (4.5) they also satisfy the relations

$$
\begin{gathered}
(-1)^{\bar{\imath} \bar{\jmath}+\bar{\imath} \bar{l}+\bar{\jmath} \bar{l}} \cdot\left[G_{i j}^{(s)}, G_{k l}^{(r)}\right]=\delta_{k j} G_{i l}^{(-s-r)}-\delta_{i l} G_{k j}^{(-s-r)}+ \\
\delta_{k,-j} G_{-i, l}^{(-s-r)} \cdot(-1)^{\bar{\imath}+\bar{\jmath}+s}-\delta_{-i, l} G_{k,-j}^{(-s-r)} \cdot(-1)^{\bar{\imath}+\bar{\jmath}+s}
\end{gathered}
$$

for all $r, s \geqslant 1$. Therefore one can define a homomorphism of the algebra $\mathrm{U}\left(\mathfrak{g}^{\prime}\right)$ onto $\operatorname{gr} Y^{*}\left(\mathfrak{q}_{N}\right)$ by

$$
u F_{j i}^{(s)} \mapsto-G_{i j}^{(s+1)} \cdot(-1)^{\bar{\imath}}
$$

But Lemma 4.2 implies that the kernel of this homomorphism is trivial

We formulate the main property of the pairing $\langle$,$\rangle as the next proposition.$

Proposition 4.4. The bilinear map $\langle\rangle:, \mathrm{Y}\left(\mathfrak{q}_{N}\right) \times \mathrm{Y}^{*}\left(\mathfrak{q}_{N}\right) \rightarrow \mathbb{C}$ is non-degenerate bi-superalgebra pairing.

Proof. Lemma 4.1 and Lemma 4.2 show that the pairing $\langle$,$\rangle is non-degenerate.$ Due to (2.19) and (4.7) the definition (4.8) implies that for any $X, Y \in \mathrm{Y}\left(\mathfrak{q}_{N}\right)$ and $X^{\prime}, Y^{\prime} \in \mathrm{Y}^{*}\left(\mathfrak{q}_{N}\right)$ we have

$$
\left\langle X Y, X^{\prime}\right\rangle=\left\langle X \otimes Y, \Delta\left(X^{\prime}\right)\right\rangle \quad \text { and } \quad\left\langle X, X^{\prime} Y^{\prime}\right\rangle=\left\langle\Delta(X), X^{\prime} \otimes Y^{\prime}\right\rangle
$$


where we employ the convention

$$
\left\langle X \otimes Y, X^{\prime} \otimes Y^{\prime}\right\rangle=\langle X, Y\rangle\left\langle X^{\prime}, Y^{\prime}\right\rangle \cdot(-1)^{\operatorname{deg} X^{\prime} \operatorname{deg} Y}
$$

for the homogeneous elements $X$ and $Y^{\prime}$. Also by definition we have $\langle 1,1\rangle=1$. Moreover, by setting $n=0$ in the definition (4.8) we get for any $s_{1}, \ldots, s_{m} \geqslant 1$

$$
\left\langle T_{i_{1} j_{1}}^{\left(s_{1}\right)} \ldots T_{i_{m} j_{m}}^{\left(s_{m}\right)}, 1\right\rangle=0, \quad m \geqslant 1 .
$$

Thus $\langle X, 1\rangle=\varepsilon(X)$ for the counit $\varepsilon$ on $\mathrm{Y}\left(\mathfrak{q}_{N}\right)$. Furthermore, by setting $m=0$ in Lemma 4.1 we obtain for any $r_{1}, \ldots, r_{n} \geqslant 1$

$$
\left\langle 1, T_{i_{1} j_{1}}^{\left(-r_{1}\right)} \ldots T_{i_{n} j_{n}}^{\left(-r_{n}\right)}\right\rangle=0, \quad n \geqslant 1 .
$$

Therefore $\left\langle 1, X^{\prime}\right\rangle=\varepsilon\left(X^{\prime}\right)$ for the counit $\varepsilon$ on $\mathrm{Y}^{*}\left(\mathfrak{q}_{N}\right)$

By Lemma 4.1 the pairing $\mathrm{Y}\left(\mathfrak{q}_{N}\right) \times \mathrm{Y}^{*}\left(\mathfrak{q}_{N}\right) \rightarrow \mathbb{C}$ extends to $\mathrm{Y}\left(\mathfrak{q}_{N}\right) \times \mathrm{Y}^{\prime}\left(\mathfrak{q}_{N}\right)$. Let us now choose any linear basis in the vector space $\mathrm{Y}\left(\mathfrak{q}_{N}\right)$. An element of this basis will be denoted by $Y_{\sigma}$. There is a system of vectors $Y^{\sigma} \in \mathrm{Y}^{\prime}\left(\mathfrak{q}_{N}\right)$ dual to this basis. The formal sum of elements from $\mathrm{Y}^{\prime}\left(\mathfrak{q}_{N}\right) \otimes \mathrm{Y}\left(\mathfrak{q}_{N}\right)$,

$$
\mathcal{R}=\sum_{\sigma} Y^{\sigma} \otimes Y_{\sigma}
$$

does not depend on the choice of basis in $\mathrm{Y}\left(\mathfrak{q}_{N}\right)$. It is called the universal $R$-matrix for the Yangian $\mathrm{Y}\left(\mathfrak{q}_{N}\right)$. The double of the Yangian is an associative complex unital algebra $\mathcal{D} Y\left(\mathfrak{q}_{N}\right)$ which contains $\mathrm{Y}\left(\mathfrak{q}_{N}\right)$ and $\mathrm{Y}^{*}\left(\mathfrak{q}_{N}\right)$ as subalgebras. Moreover, it is generated by these two subalgebras. We also impose the relations

$$
\mathcal{R} \cdot \Delta(X)=\Delta^{\circ}(X) \cdot \mathcal{R}, \quad X \in \mathrm{Y}^{*}\left(\mathfrak{q}_{N}\right)
$$

where $\Delta^{\circ}$ is composition of the comultiplications $\Delta$ on $\mathrm{Y}^{*}\left(\mathfrak{q}_{N}\right)$ with the involutive automorphism $\theta$ of the algebra $\mathrm{Y}^{*}\left(\mathfrak{q}_{N}\right) \otimes \mathrm{Y}^{*}\left(\mathfrak{q}_{N}\right)$. Either side of the equality (4.16) makes sense as a formal sum of elements from $\mathrm{Y}^{\prime}\left(\mathfrak{q}_{N}\right) \otimes \mathcal{D} Y\left(\mathfrak{q}_{N}\right)$.

The equalities (4.15) imply that for the comultiplications $\Delta$ on $\mathrm{Y}\left(\mathfrak{q}_{N}\right), \mathrm{Y}^{\prime}\left(\mathfrak{q}_{N}\right)$

$$
\Delta \otimes \operatorname{id}(\mathcal{R})=\mathcal{R}_{13} \mathcal{R}_{23}, \quad \operatorname{id} \otimes \Delta(\mathcal{R})=\mathcal{R}_{12} \mathcal{R}_{13}
$$

where

$$
\mathcal{R}_{12}=\sum_{\sigma} Y^{\sigma} \otimes Y_{\sigma} \otimes 1, \quad \mathcal{R}_{13}=\sum_{\sigma} Y^{\sigma} \otimes 1 \otimes Y_{\sigma}, \quad \mathcal{R}_{23}=\sum_{\sigma} 1 \otimes Y^{\sigma} \otimes Y_{\sigma} .
$$

It follows from $(4.17)$ that $\mathcal{R}^{-1}=\mathrm{id} \otimes S(\mathcal{R})$ for the antipodal map $S$ on $\mathrm{Y}\left(\mathfrak{q}_{N}\right)$.

Let us now regard the parameter $z$ in the definition (2.20) as a formal parameter. Then we get a representation $\mathrm{Y}\left(\mathfrak{q}_{N}\right) \rightarrow \operatorname{End}\left(\mathbb{C}^{N \mid N}\right)[z]$. We will denote it by $\rho_{z}$. Moreover, by comparing $(2.8),(2.11)$ to $(4.3),(4.4)$ respectively we obtain that

$$
\mathrm{Y}^{*}\left(\mathfrak{q}_{N}\right) \otimes \operatorname{End}\left(\mathbb{C}^{N \mid N}\right)[[v]] \rightarrow \operatorname{End}\left(\mathbb{C}^{N \mid N}\right)^{\otimes 2}\left[\left[z^{-1}, v\right]\right]: T^{*}(v) \mapsto R(z, v)
$$

determines a representation $\mathrm{Y}^{*}\left(\mathfrak{q}_{N}\right) \rightarrow \operatorname{End}\left(\mathbb{C}^{N \mid N}\right)\left[z^{-1}\right]$. We will denote it by $\rho_{z}^{*}$. More explicitly, for each index $s \geqslant 1$ we have

$$
\rho_{z}^{*}: T_{i j}^{(-s)} \mapsto-\left(E_{j i} z^{-s}+E_{-j,-i}(-z)^{-s}\right) \cdot(-1)^{\bar{\imath}} .
$$

Therefore we can now extend $\rho_{z}^{*}$ to a representation $\mathrm{Y}^{\prime}\left(\mathfrak{q}_{N}\right) \rightarrow \operatorname{End}\left(\mathbb{C}^{N \mid N}\right)\left[\left[z^{-1}\right]\right]$. 
Proposition 4.5. We have $\rho_{z}^{*} \otimes \operatorname{id}(\mathcal{R})=T(z)$ and also id $\otimes \rho_{z}(\mathcal{R})=T^{*}(z)$.

Proof. By the definition of our canonical pairing $\mathrm{Y}\left(\mathfrak{q}_{N}\right) \otimes \mathrm{Y}^{*}\left(\mathfrak{q}_{N}\right) \rightarrow \mathbb{C}$, for any $m \geqslant 0$ the element $T^{*}(z) \in \mathrm{Y}^{*}\left(\mathfrak{q}_{N}\right) \otimes \operatorname{End}\left(\mathbb{C}^{N \mid N}\right)[[z]]$ has the property that

$$
\begin{gathered}
\operatorname{End}\left(\mathbb{C}^{N \mid N}\right)^{\otimes m} \otimes \mathrm{Y}\left(\mathfrak{q}_{N}\right) \otimes \mathrm{Y}^{*}\left(\mathfrak{q}_{N}\right) \otimes \operatorname{End}\left(\mathbb{C}^{N \mid N}\right) \rightarrow \operatorname{End}\left(\mathbb{C}^{N \mid N}\right)^{\otimes(m+1)}: \\
T_{1}\left(u_{1}\right) \ldots T_{m}\left(u_{m}\right) \otimes T^{*}(z) \mapsto R_{1, m+1}\left(u_{1}, z\right) \ldots R_{m, m+1}\left(u_{m}, z\right)
\end{gathered}
$$

under the map id $\otimes \beta \otimes$ id. To get the second equality in Proposition 4.5 it suffuces to show that the element id $\otimes \rho_{z}(\mathcal{R}) \in \mathrm{Y}^{\prime}\left(\mathfrak{q}_{N}\right) \otimes \operatorname{End}\left(\mathbb{C}^{N \mid N}\right)[z]$ has the same property. Due to the definition of the element $\mathcal{R}$ the latter property amounts to

$$
\begin{array}{r}
\operatorname{id} \otimes \rho_{z}: \operatorname{End}\left(\mathbb{C}^{N \mid N}\right)^{\otimes m} \otimes \mathrm{Y}\left(\mathfrak{q}_{N}\right) \rightarrow \operatorname{End}\left(\mathbb{C}^{N \mid N}\right)^{\otimes m+1}: \\
T_{1}\left(u_{1}\right) \ldots T_{m}\left(u_{m}\right) \mapsto R_{1, m+1}\left(u_{1}, z\right) \ldots R_{m, m+1}\left(u_{m}, z\right)
\end{array}
$$

which holds by (2.20). Proof of the first equality in Proposition 4.5 is similar

Corollary 4.6. Representations $\rho_{z}$ of $\mathrm{Y}\left(\mathfrak{q}_{N}\right)$ and $\rho_{z}^{*}$ of $\mathrm{Y}^{*}\left(\mathfrak{q}_{N}\right)$ determine a representation of the algebra $\mathcal{D Y}\left(\mathfrak{q}_{N}\right)$ in $\operatorname{End}\left(\mathbb{C}^{N \mid N}\right)\left[z, z^{-1}\right]$.

Proof. According to (4.16) we have to verify for any $X \in \mathrm{Y}^{*}\left(\mathfrak{q}_{N}\right)$ the relation

$$
\left(\operatorname{id} \otimes \rho_{u}(\mathcal{R})\right) \cdot\left(\operatorname{id} \otimes \rho_{u}^{*}(\Delta(X))\right)=\left(\operatorname{id} \otimes \rho_{u}^{*}\left(\Delta^{\prime}(X)\right)\right) \cdot\left(\operatorname{id} \otimes \rho_{u}(\mathcal{R})\right)
$$

in $Y^{\prime}\left(\mathfrak{q}_{N}\right) \otimes \operatorname{End}\left(\mathbb{C}^{N \mid N}\right)\left[u, u^{-1}\right]$. It suffices to set here $X=T_{i j}^{*}(v)$. Due to the definitions (4.7) and (4.18) the collection of the resulting relations for all indices $i, j$ is exactly the defining relation (4.3)

To write down commutation relations in the algebra $\mathcal{D Y}\left(\mathfrak{q}_{N}\right)$ we will use the tensor product $\operatorname{End}\left(\mathbb{C}^{N \mid N}\right) \otimes \mathcal{D Y}\left(\mathfrak{q}_{N}\right) \otimes \operatorname{End}\left(\mathbb{C}^{N \mid N}\right)$. There is a natural embedding of the algebra End $\left(\mathbb{C}^{N \mid N}\right)^{\otimes 2}$ into this tensor product: $X \otimes Y \mapsto X \otimes 1 \otimes Y$ for any elements $X, Y \in$ End $\left(\mathbb{C}^{N \mid N}\right)$. Denote by $\widehat{R}(u, v)$ the image of $(2.6)$ with respect to this embedding. Then we obtain another corollary to Proposition 4.5.

Corollary 4.7. In $\operatorname{End}\left(\mathbb{C}^{N \mid N}\right) \otimes \mathcal{D Y}\left(\mathfrak{q}_{N}\right) \otimes \operatorname{End}\left(\mathbb{C}^{N \mid N}\right)\left[\left[u^{-1}, v\right]\right]$ we have

$$
(T(u) \otimes 1) \cdot \widehat{R}(u, v) \cdot\left(1 \otimes T^{*}(v)\right)=\left(1 \otimes T^{*}(v)\right) \cdot \widehat{R}(u, v) \cdot(T(u) \otimes 1) .
$$

Proof. Put $X=T_{i j}^{*}(v)$ in (4.16). Apply the homomorphism $\rho_{u}^{*} \otimes$ id to the resulting equality and use the definition (4.7). Then we get the equality

$$
\sum_{k} T(u) \cdot\left(\rho_{u}^{*}\left(T_{i k}^{*}(v)\right) \otimes T_{k j}^{*}(v)\right) \cdot(-1)^{(\bar{\imath}+\bar{k})(\bar{\jmath}+\bar{k})}=\sum_{k}\left(\rho_{u}^{*}\left(T_{k j}^{*}(v)\right) \otimes T_{i k}^{*}(v)\right) \cdot T(u)
$$

in $\operatorname{End}\left(\mathbb{C}^{N \mid N}\right) \otimes \mathcal{D Y}\left(\mathfrak{q}_{N}\right)\left[\left[u^{-1}, v\right]\right]$ by Proposition 4.5. Due to the definition (4.18) the collection of the above equalities for all indices $i, j$ is equivalent to (4.19) 
Theorem 4.8. The relation (4.19) implies the defining relations (4.16).

Proof. Let $u_{1}, u_{2}, \ldots$ be independent formal parameters. For each $n=1,2, \ldots$ take the tensor product $\nu^{*}$ of the representations $\rho_{z}^{*}: \mathrm{Y}^{\prime}\left(\mathfrak{q}_{N}\right) \rightarrow \operatorname{End}\left(\mathbb{C}^{N \mid N}\right)\left[\left[z^{-1}\right]\right]$ with $z=u_{1}, \ldots, u_{n}$. Using our descending $\mathbb{Z}$-filtration on the algebra $\mathrm{Y}^{*}\left(\mathfrak{q}_{N}\right)$ and Corollary 4.3 , we can prove that the kernels of all representations $\nu^{*}$ have zero intersection. The proof is similar to the proof of Proposition 2.2 and is omitted here. Hence it suffices to derive from the relation (4.19) that for any $X \in \mathrm{Y}^{*}\left(\mathfrak{q}_{N}\right)$

$$
\nu^{*} \otimes \operatorname{id}(\mathcal{R} \cdot \Delta(X))=\nu^{*} \otimes \operatorname{id}\left(\Delta^{\circ}(X) \cdot \mathcal{R}\right)
$$

Let us again use Proposition 4.5 along with the definition (4.7). The collection of all equalities (4.20) for $X=T_{i j}^{*}(v)$ with various indices $i, j$ can be written as the single relation in the algebra $\operatorname{End}\left(\mathbb{C}^{N \mid N}\right)^{\otimes n} \otimes \mathcal{D} Y\left(\mathfrak{q}_{N}\right) \otimes \operatorname{End}\left(\mathbb{C}^{N \mid N}\right)\left[\left[u_{1}^{-1}, \ldots, u_{n}^{-1}, v\right]\right]$

$$
\begin{array}{r}
\left(T_{1}\left(u_{1}\right) \ldots T_{n}\left(u_{n}\right) \otimes 1\right) \cdot \widehat{R}_{1, n+1}\left(u_{1}, v\right) \ldots \widehat{R}_{n, n+1}\left(u_{n}, v\right) \cdot\left(1 \otimes T^{*}(v)\right)= \\
\left(1 \otimes T^{*}(v)\right) \cdot \widehat{R}_{1, n+1}\left(u_{1}, v\right) \ldots \widehat{R}_{n, n+1}\left(u_{n}, v\right) \cdot\left(T_{1}\left(u_{1}\right) \ldots T_{n}\left(u_{n}\right) \otimes 1\right)
\end{array}
$$

where $\widehat{R}_{1, n+1}\left(u_{1}, v\right), \ldots, \widehat{R}_{n, n+1}\left(u_{n}, v\right)$ are respectively the images of the elements

$$
R_{1, n+1}\left(u_{1}, v\right), \ldots, R_{n, n+1}\left(u_{n}, v\right) \in \operatorname{End}\left(\mathbb{C}^{N \mid N}\right)^{\otimes(n+1)}\left[\left[u_{1}^{-1}, \ldots, u_{n}^{-1}, v\right]\right]
$$

under the natural embedding of the latter algebra to the former one. But using (4.19) repeatedly, we obtain (4.21)

Thus we have proved that the relations (4.19) together with the relations $(2.7),(2.9)$ and $(4.3),(4.4)$ are defining relations for the algebra $\mathcal{D Y}\left(\mathfrak{q}_{N}\right)$; cf. [KT, Section 2].

\section{Representations of the Yangian}

Here we construct a wide class of irreducible representations of the algebra $\mathrm{Y}\left(\mathfrak{q}_{N}\right)$, by using irreducible represenations of a certain less complicated algebra $\mathcal{A}_{n}$ where $n=1,2, \ldots$ is arbitrary. The algebra $\mathcal{A}_{n}$ was introduced in [N2] and called the degenerate affine Sergeev algebra, in honour of the author of [S1,S2]. This is an analogue of the degenerate affine Hecke algebra, which was employed in [D2] to construct irreducible representations of the Yangian $\mathrm{Y}\left(\mathfrak{g l}_{N}\right)$ of the general linear Lie algebra $\mathfrak{g l}_{N}$. Results presented in this section were reported for the first time in the summer of 1991 at the Wigner Symposium in Goslar, Germany. They were also reported in the autumn of 1992 at the Symposium on Representation Theory in Yamagata, Japan. Non-degenerate affine Sergeev algebra is defined in [JN], cf. [O2].

Consider the crossed product $H_{n}$ of the symmetric group $S_{n}$ with the Clifford algebra over the complex field $\mathbb{C}$ on $n$ anticommuting generators. These generators are denoted by $c_{1}, \ldots, c_{n}$ and are subjected to the relations

$$
c_{p}^{2}=-1, \quad c_{p} c_{q}=-c_{q} c_{p} \quad \text { if } \quad p \neq q .
$$

The group $S_{n}$ acts on the Clifford algebra by permutations of these $n$ generators. Let $w_{p q} \in S_{n}$ be the transposition of two numbers $p \neq q$. There is a representation $H_{n} \rightarrow \operatorname{End}\left(\mathbb{C}^{N \mid N}\right)^{\otimes n}$ determined by the assignments $w_{p q} \mapsto P_{p q}$ and $c_{p} \mapsto J_{p}$, see definitions (1.8) and (2.5). The supercommutant of the image of this representation 
in $\operatorname{End}\left(\mathbb{C}^{N \mid N}\right)^{\otimes n}$ coincides by [S2, Theorem 3] with the image of the $n$-th tensor power of defining representation $\mathrm{U}\left(\mathfrak{q}_{N}\right) \rightarrow \operatorname{End}\left(\mathbb{C}^{N \mid N}\right)$. By definition, the complex algebra $\mathcal{A}_{n}$ is generated by the algebra $H_{n}$ and the pairwise commuting elements $x_{1}, \ldots, x_{n}$ with the following relations:

$$
\begin{gathered}
x_{p} w_{q, q+1}=w_{q, q+1} x_{p} \quad \text { if } \quad p \neq q, q+1 \\
x_{p} w_{p, p+1}=w_{p, p+1} x_{p+1}-1-c_{p} c_{p+1} ; \\
x_{p} c_{q}=c_{q} x_{p} \quad \text { if } \quad p \neq q, \quad x_{p} c_{p}=-c_{p} x_{p} .
\end{gathered}
$$

The algebra $\mathcal{A}_{n}$ is $\mathbb{Z}_{2}$-graded so that $\operatorname{deg} c_{p}=1$ while $\operatorname{deg} x_{p}=\operatorname{deg} w_{p q}=0$.

Proposition 5.1. Let $Y$ range over a basis in $H_{n}$ and let each of $s_{1}, \ldots, s_{n}$ range over the non-negative integers. Then the products $Y x_{1}^{s_{1}} \ldots x_{n}^{s_{n}}$ form a basis in $\mathcal{A}_{n}$. Proof. For $m=0,1,2, \ldots$ one can define a homomorphism $\gamma_{m}: \mathcal{A}_{n} \rightarrow H_{m+n}$ by

$$
\gamma_{m}: \quad w_{p q} \mapsto w_{m+p, m+q}, \quad c_{p} \mapsto c_{m+p}, \quad x_{p} \mapsto \sum_{1 \leqslant r<m+p}\left(1+c_{m+p} c_{r}\right) w_{m+p, r} .
$$

This can be verified directly by (5.1). Suppose that $m \geqslant s_{1}+\ldots+s_{n}$. Choose for every $p=1, \ldots, n$ a subsequence $\mathcal{M}_{p}$ in $1, \ldots, m$ of cardinality $s_{p}$ so that all these subsequences are disjoint. Write the image of $x_{1}^{s_{1}} \ldots x_{n}^{s_{n}}$ under $\gamma_{m}$ as a linear combination of the elements $c_{r} \ldots c_{r^{\prime}} w \in H_{m+n}$ where $1 \leqslant r<\ldots<r^{\prime} \leqslant m+n$ and $w \in S_{m+n}$. Consider the terms in this linear combination where $w$ has the maximal possible length. Amongst them we find the term

$$
\prod_{1 \leqslant p \leqslant n}\left(\prod_{r \in \mathcal{M}_{p}} w_{m+p, r}\right)
$$

which allows us to restore the exponents $s_{1}, \ldots, s_{n}$ and the basis element $Y \in H_{n}$ from the image $\gamma_{m}\left(Y x_{1}^{s_{1}} \ldots x_{n}^{s_{n}}\right)$ uniquely.

By using the relations (5.1) every element of the algebra $\mathcal{A}_{n}$ can be expressed as a finite linear combination of the products $Y x_{1}^{s_{1}} \ldots x_{n}^{s_{n}}$. Now take any such a linear combination and suppose that for all its terms $m \geqslant s_{1}+\ldots+s_{n}$. Then the above analysis shows that for all the terms, the images $\gamma_{m}\left(Y x_{1}^{s_{1}} \ldots x_{n}^{s_{n}}\right)$ are linearly independent in $H_{m+n}$

Along with pairwise commuting generators $x_{1}, \ldots, x_{n}$ we need the non-commuting generators

$$
y_{p}=x_{p}-\sum_{1 \leqslant q<p}\left(1+c_{p} c_{q}\right) w_{p q} ; \quad p=1, \ldots, n .
$$

Observe that the generators $y_{1}, \ldots, y_{n}$ belong to the kernel of the homomorphism $\gamma_{0}: \mathcal{A}_{n} \rightarrow H_{n}$ as defined in the proof of Proposition 5.1. By using this observation,

$$
\begin{gathered}
w y_{p} w^{-1}=y_{w(p)}, \quad w \in S_{n} ; \\
y_{p} c_{q}=c_{q} y_{p} \text { if } p \neq q, \quad y_{p} c_{p}=-c_{p} y_{p} .
\end{gathered}
$$

Relations (5.1) and relations in the first line of (5.2) yield the commutation relations

$$
w_{p q}\left[y_{p}, y_{q}\right]=y_{p}-y_{q}+c_{p} c_{q}\left(y_{p}+y_{q}\right)
$$

for the generators $y_{p}, y_{q}$ with arbitrary indices $p, q=1, \ldots, n$. 
Now take the tensor product of the $\mathbb{Z}_{2}$-graded algebras End $\left(\mathbb{C}^{N \mid N}\right)^{\otimes n}$ and $\mathcal{A}_{n}$. Since the elements $x_{1}, \ldots, x_{n} \in \mathcal{A}_{n}$ pairwise commute, the assignment

$$
\operatorname{End}\left(\mathbb{C}^{N \mid N}\right) \otimes \mathrm{Y}\left(\mathfrak{q}_{N}\right)\left[\left[u^{-1}\right]\right] \rightarrow \operatorname{End}\left(\mathbb{C}^{N \mid N}\right)^{\otimes(n+1)} \otimes \mathcal{A}_{n}\left[\left[u^{-1}\right]\right]: \quad T(u) \mapsto
$$

$$
\prod_{1 \leqslant p \leqslant n}^{\rightarrow}\left(1-P_{1, p+1} \otimes \frac{1}{u-x_{p}}+P_{1, p+1} J_{1} J_{p+1} \otimes \frac{1}{u+x_{p}}\right)
$$

determines a homomorphism $\mathrm{Y}\left(\mathfrak{q}_{N}\right) \rightarrow \operatorname{End}\left(\mathbb{C}^{N \mid N}\right)^{\otimes n} \otimes \mathcal{A}_{n}$, see (2.6) and (2.22). As usual, the fractions $1 /\left(u \pm x_{p}\right)$ in (5.3) should be expanded as formal power series in $u^{-1}$. The next proposition is a key to our construction, cf. [BGHP, Section 2.1].

Proposition 5.2. a) The difference between the product (5.3) and the sum

$$
1-\sum_{1 \leqslant p \leqslant n} P_{1, p+1} \otimes \frac{1}{u-y_{p}}+\sum_{1 \leqslant p \leqslant n} P_{1, p+1} J_{1} J_{p+1} \otimes \frac{1}{u+y_{p}}
$$

belongs to the left ideal in the algebra $\operatorname{End}\left(\mathbb{C}^{N \mid N}\right)^{\otimes(n+1)} \otimes \mathcal{A}_{n}\left[\left[u^{-1}\right]\right]$ generated by all the elements $1-P_{p+1, q+1} \otimes w_{p q}$ and $1-J_{p+1} J_{q+1} \otimes c_{p} c_{q}$ with $p \neq q$.

b) The sum (5.4) commutes with the elements $P_{p+1, q+1} \otimes w_{p q}$ and $J_{p+1} \otimes c_{p}$.

Proof. Part (b) immediately follows from the relations (5.2). To prove (a), we will use induction on $n$. When $n=1$, the equality $x_{1}=y_{1}$ provides the induction base. Suppose that $n>1$ and that Proposition 5.2 is true for $n-1$ instead of $n$. Then the difference between (5.3) and (5.4) equals

$$
\begin{aligned}
-(1- & \left.\sum_{1 \leqslant p<n} P_{1, p+1} \otimes \frac{1}{u-y_{p}}+\sum_{1 \leqslant p<n} P_{1, p+1} J_{1} J_{p+1} \otimes \frac{1}{u+y_{p}}\right) \times \\
& \left(P_{1, n+1} \otimes \frac{1}{u-x_{n}}-P_{1, n+1} J_{1} J_{n+1} \otimes \frac{1}{u+x_{n}}\right)+ \\
& \left(P_{1, n+1} \otimes \frac{1}{u-y_{n}}-P_{1, n+1} J_{1} J_{n+1} \otimes \frac{1}{u+y_{n}}\right) .
\end{aligned}
$$

Up to the terms divisible on the right by $1-P_{p+1, n+1} \otimes w_{p n}$ or $1-J_{p+1} J_{n+1} \otimes c_{p} c_{n}$ with $1 \leqslant p<n$, the above sum equals

$$
\begin{gathered}
P_{1, n+1} \otimes\left(\frac{1}{u-y_{n}}-\frac{1}{u-x_{n}}+\sum_{1 \leqslant p<n} \frac{1}{u-x_{n}}\left(\frac{1}{u-y_{p}}+\frac{1}{u+y_{p}} c_{n} c_{p}\right) w_{p n}\right)+ \\
P_{1, n+1} J_{1} J_{n+1} \otimes\left(\frac{1}{u+x_{n}}-\frac{1}{u+y_{n}}+\sum_{1 \leqslant p<n} \frac{1}{u+x_{n}}\left(\frac{1}{u+y_{p}}+\frac{1}{u-y_{p}} c_{n} c_{p}\right) w_{p n}\right)
\end{gathered}
$$

where we have used the fact that $x_{n}$ commutes with $y_{1}, \ldots, y_{n-1}$. In these two lines, the second tensor factors differ by changing $u$ to $-u$. It suffices to show that in the first line, the second tensor factor equals zero in $\mathcal{A}_{n}\left[\left[u^{-1}\right]\right]$. Multiplying this tensor factor on the left by $u-x_{n}$, on the right by $u-y_{n}$, and using the relations (5.2) in $\mathcal{A}_{n}$ we get the sum

$$
\left(u-x_{n}\right)-\left(u-y_{n}\right)+\sum_{1 \leqslant p<n}\left(1+c_{n} c_{p}\right) w_{p n} .
$$

But this sum equals zero by the definition of the element $y_{n} \in \mathcal{A}_{n}$ 
Take any representation $\xi: \mathcal{A}_{n} \rightarrow \operatorname{End}(U)$ where the complex vector space $U$ is $\mathbb{Z}_{2}$-graded, but not necessary finite-dimensional. The algebra $\operatorname{End}(U)$ is then $\mathbb{Z}_{2}$-graded. We assume that the homomorphism $\xi$ preserves $\mathbb{Z}_{2}$-gradation.

Take the tensor product of vector spaces $\left(\mathbb{C}^{N \mid N}\right)^{\otimes n} \otimes U$. We identify the tensor product End $\left(\mathbb{C}^{N \mid N}\right)^{\otimes n} \otimes \operatorname{End}(U)$ with the algebra End $\left(\left(\mathbb{C}^{N \mid N}\right)^{\otimes n} \otimes U\right)$ so that

$$
(A \otimes B) \cdot(a \otimes b)=(A a) \otimes(B b) \cdot(-1)^{\operatorname{deg} a \operatorname{deg} B} .
$$

for any homogeneous $a \in\left(\mathbb{C}^{N \mid N}\right)^{\otimes n}, b \in U$ and $A \in \operatorname{End}\left(\mathbb{C}^{N \mid N}\right)^{\otimes n}, B \in \operatorname{End}(U)$. There is an action of the hyperoctahedral group $S_{n} \ltimes \mathbb{Z}_{2}^{n}$ in $\left(\mathbb{C}^{N \mid N}\right)^{\otimes n} \otimes U$. The transposition $w_{p q} \in S_{n}$ acts as the operator $P_{p q} \otimes \xi\left(w_{p q}\right)$ while the generator of $p$-th direct factor $\mathbb{Z}_{2}$ in $\mathbb{Z}_{2}^{n}$ acts as the operator $J_{p} \otimes \xi\left(c_{p}\right) \cdot \sqrt{-1}$. Denote by $\alpha$ this action. Let $V$ be the space of co-invariants with respect to $\alpha$. This is the quotient space of $\left(\mathbb{C}^{N \mid N}\right)^{\otimes n} \otimes U$ with respect to the subspace spanned by the images of all the operators $\alpha(g)-1$ with $g \in S_{n} \ltimes \mathbb{Z}_{2}^{n}$. Each of these operators has $\mathbb{Z}_{2}$-degree zero, therefore the vector space $V$ inherits $\mathbb{Z}_{2}$-gradation from $\left(\mathbb{C}^{N \mid N}\right)^{\otimes n} \otimes U$.

Let us expand the element (5.4) of $\operatorname{End}\left(\mathbb{C}^{N \mid N}\right)^{\otimes(n+1)} \otimes \mathcal{A}_{n}\left[\left[u^{-1}\right]\right]$ relative to the basis of standard matrix units in the first tensor factor $\operatorname{End}\left(\mathbb{C}^{N \mid N}\right)$. Then for $s=0,1,2, \ldots$ the coefficient in $(5.4)$ at $E_{i j} u^{-s-1} \in \operatorname{End}\left(\mathbb{C}^{N \mid N}\right)\left[\left[u^{-1}\right]\right]$ is

$$
\sum_{1 \leqslant p \leqslant n}\left(\iota_{p}\left(E_{j i}\right)+(-1)^{s} \iota_{p}\left(E_{-j,-i}\right)\right) \otimes y_{p}^{s} \cdot(-1)^{\bar{\jmath}+1} \in \operatorname{End}\left(\mathbb{C}^{N \mid N}\right)^{\otimes n} \otimes \mathcal{A}_{n}
$$

where $\iota_{p}$ denotes embedding of $\operatorname{End}\left(\mathbb{C}^{N \mid N}\right)$ to $\operatorname{End}\left(\mathbb{C}^{N \mid N}\right)^{\otimes n}$ as the $p$-th tensor factor. All these coefficients commute with the elements $P_{p q} \otimes w_{p q}$ and $J_{p} \otimes c_{p}$ in the algebra $\operatorname{End}\left(\mathbb{C}^{N \mid N}\right)^{\otimes n} \otimes \mathcal{A}_{n}$ by part (b) of Proposition 5.2. By part (a), one can now define a representation of the $\mathbb{Z}_{2}$-graded algebra $\mathrm{Y}\left(\mathfrak{q}_{N}\right)$ in $V$ by assigning to the generator $T_{i j}^{(s+1)}$ with $s \geqslant 0$ the action, induced in $V$ by the operator

$$
\sum_{1 \leqslant p \leqslant n}\left(\iota_{p}\left(E_{j i}\right)+(-1)^{s} \iota_{p}\left(E_{-j,-i}\right)\right) \otimes \xi\left(y_{p}^{s}\right) \cdot(-1)^{\bar{\jmath}+1}
$$

in the space $\left(\mathbb{C}^{N \mid N}\right)^{\otimes n} \otimes U$. Correspondence $U \mapsto V$ is the $\mathrm{Y}\left(\mathfrak{q}_{N}\right)$-analogue of the Drinfeld functor [D2] for the Yangian $\mathrm{Y}\left(\mathfrak{g l}_{N}\right)$. Denote our correspondence by $\mathcal{F}_{N}$.

For any positive integer $n^{\prime}$ consider the tensor product $\mathcal{A}_{n} \otimes \mathcal{A}_{n^{\prime}}$ of $\mathbb{Z}_{2}$-graded algebras. It is isomorphic to the subalgebra in $\mathcal{A}_{n+n^{\prime}}$ generated by transpositions $w_{p q}$ where $1 \leqslant p<q \leqslant n$ or $n+1 \leqslant p<q \leqslant n+n^{\prime}$, along with all the elements $c_{p}$ and $x_{p}$ where $1 \leqslant p \leqslant n+n^{\prime}$. Take any $\mathbb{Z}_{2}$-graded representation $\xi^{\prime}: \mathcal{A}_{n^{\prime}} \rightarrow \operatorname{End}\left(U^{\prime}\right)$. Consider the representation of the algebra $\mathcal{A}_{n+n^{\prime}}$ induced from the representation of $\mathcal{A}_{n} \otimes \mathcal{A}_{n^{\prime}}$ in $U \otimes U^{\prime}$. The algebra $\mathcal{A}_{n+n^{\prime}}$ acts in the vector space $\mathcal{A}_{n+n^{\prime}} \otimes U \otimes U^{\prime}$ via left multiplication at the first tensor factor. We realise the induced representation in the quotient space of $\mathcal{A}_{n+n^{\prime}} \otimes U \otimes U^{\prime}$ by the following relations: for homogeneous $b \in U, b^{\prime} \in U^{\prime}, X \in \mathcal{A}_{n+n^{\prime}}, Y \in \mathcal{A}_{n}, Y^{\prime} \in \mathcal{A}_{n^{\prime}}$

$$
(X Z) \otimes b \otimes b^{\prime}=X \otimes(\xi(Y) b) \otimes\left(\xi^{\prime}\left(Y^{\prime}\right) b^{\prime}\right) \cdot(-1)^{\operatorname{deg} b \operatorname{deg} Y^{\prime}}
$$

where $Z$ stands for the image of $Y \otimes Y^{\prime} \in \mathcal{A}_{n} \otimes \mathcal{A}_{n^{\prime}}$ in the algebra $\mathcal{A}_{n+n^{\prime}}$. Let us denote by $U \odot U^{\prime}$ this quotient space.

Consider the representation of the algebra $\mathrm{Y}\left(\mathfrak{q}_{N}\right)$ in the space $V^{\prime}=\mathcal{F}_{N}\left(U^{\prime}\right)$. Determine a representation of $\mathrm{Y}\left(\mathfrak{q}_{N}\right)$ in $V \otimes V^{\prime}$ using the comultiplication (2.19). 
Proposition 5.3. Representation of the algebra $\mathrm{Y}\left(\mathfrak{q}_{N}\right)$ corresponding to $U \odot U^{\prime}$, is equivalent to the representation of $\mathrm{Y}\left(\mathfrak{q}_{N}\right)$ in $V \otimes V^{\prime}$.

Proof. By definition, $V \otimes V^{\prime}$ is a quotient space of $\left(\mathbb{C}^{N \mid N}\right)^{\otimes n} \otimes U \otimes\left(\mathbb{C}^{N \mid N}\right)^{\otimes n^{\prime}} \otimes U^{\prime}$. Identify the latter tensor product with $\left(\mathbb{C}^{N \mid N}\right)^{\otimes\left(n+n^{\prime}\right)} \otimes U \otimes U^{\prime}$ via the linear map

$$
a \otimes b \otimes a^{\prime} \otimes b^{\prime} \mapsto a \otimes a^{\prime} \otimes b \otimes b^{\prime} \cdot(-1)^{\operatorname{deg} a^{\prime} \operatorname{deg} b} .
$$

Let $W$ be the quotient space of $\left(\mathbb{C}^{N \mid N}\right)^{\otimes\left(n+n^{\prime}\right)} \otimes U \otimes U^{\prime}$ corresponding to $V \otimes V^{\prime}$. To determine the action of the algebra $\mathrm{Y}\left(\mathfrak{q}_{N}\right)$ in $W$, we can use the representation $\mathrm{Y}\left(\mathfrak{q}_{N}\right) \rightarrow \operatorname{End}\left(\mathbb{C}^{N \mid N}\right)^{\otimes\left(n+n^{\prime}\right)} \otimes \operatorname{End}(U) \otimes \operatorname{End}\left(U^{\prime}\right)$, with respect to which the element $T(u) \in \operatorname{End}\left(\mathbb{C}^{N \mid N}\right) \otimes \mathrm{Y}\left(\mathfrak{q}_{N}\right)\left[\left[u^{-1}\right]\right]$ is represented by the product

$$
\begin{aligned}
& \prod_{1 \leqslant p \leqslant n}^{\rightarrow}\left(1-P_{1, p+1} \otimes \frac{1}{u-\xi\left(x_{p}\right) \otimes 1}+P_{1, p+1} J_{1} J_{p+1} \otimes \frac{1}{u+\xi\left(x_{p}\right) \otimes 1}\right) \times \\
& \prod_{1 \leqslant q \leqslant n^{\prime}}^{\rightarrow}\left(1-P_{1, n+q+1} \otimes \frac{1}{u-1 \otimes \xi^{\prime}\left(x_{q}\right)}+P_{1, n+q+1} J_{1} J_{n+q+1} \otimes \frac{1}{u+1 \otimes \xi^{\prime}\left(x_{q}\right)}\right)
\end{aligned}
$$

in the algebra $\operatorname{End}\left(\mathbb{C}^{N \mid N}\right)^{\otimes\left(n+n^{\prime}+1\right)} \otimes \operatorname{End}(U) \otimes \operatorname{End}\left(U^{\prime}\right)$. Here we used (2.19).

The space of the representation of $\mathrm{Y}\left(\mathfrak{q}_{N}\right)$ corresponding to $U \odot U^{\prime}$, is a quotient space of $\left(\mathbb{C}^{N \mid N}\right)^{\otimes\left(n+n^{\prime}\right)} \otimes \mathcal{A}_{n+n^{\prime}} \otimes U \otimes U^{\prime}$. The assignment

$$
a \otimes a^{\prime} \otimes b \otimes b^{\prime} \mapsto a \otimes a^{\prime} \otimes 1 \otimes b \otimes b^{\prime} \in\left(\mathbb{C}^{N \mid N}\right)^{\otimes\left(n+n^{\prime}\right)} \otimes \mathcal{A}_{n+n^{\prime}} \otimes U \otimes U^{\prime}
$$

induces an isomorphism of $W$ to this quotient. This isomorphism commutes with the action of the algebra $\mathrm{Y}\left(\mathfrak{q}_{N}\right)$, since by (5.6) for any $1 \leqslant p \leqslant n$ and $1 \leqslant q \leqslant n^{\prime}$, the actions of $x_{p}$ and $x_{n+q}$ on the class of $1 \otimes b \otimes b^{\prime}$ in $U \odot U^{\prime}$ yield the classes of $1 \otimes \xi\left(x_{p}\right) b \otimes b^{\prime}$ and $1 \otimes b \otimes \xi^{\prime}\left(b^{\prime}\right)$ respectively

To give an example of the correspondence $\mathcal{F}_{N}: U \mapsto V$, consider any principal series representation of the algebra $\mathcal{A}_{n}$. This is the representation induced from a character $\chi$ of the commutative subalgebra in $\mathcal{A}_{n}$ generated by $x_{1}, \ldots, x_{n}$. Note that this subalgebra is maximal commutative by [N2,Proposition 3.1]. Take character $\chi$ such that $\chi\left(x_{1}\right)=z_{1}, \ldots, \chi\left(x_{n}\right)=z_{n}$ in $\mathbb{C}$. Due to Proposition 5.1, the space $U_{z_{1} \ldots z_{n}}$ of the corresponding principal series representation of $\mathcal{A}_{n}$ is identified with algebra $H_{n}$, which acts on itself via left multiplication. The action of $x_{p} \in \mathcal{A}_{n}$ is then uniquely determined by the assignment $1 \mapsto z_{p}$ in the space $H_{n}$.

Corollary 5.4. The representation of the algebra $\mathrm{Y}\left(\mathfrak{q}_{N}\right)$ corresponding to $U_{z_{1} \ldots z_{n}}$ is equivalent to the representation (2.22).

Proof. The representations of the algebra $\mathcal{A}_{n}$ in $U_{z_{1} \ldots z_{n}}$ and $U_{z_{1}} \odot \ldots \odot U_{z_{n}}$ are equivalent. Due to Proposition 5.3 it suffices to consider the case $n=1$. The space of the representation of $\mathrm{Y}\left(\mathfrak{q}_{N}\right)$ corresponding to $U_{z}$ is the quotient of $\mathbb{C}^{N \mid N} \otimes H_{1}$ by the relations

$$
a \otimes c_{1} \cdot \sqrt{-1}=(J a) \otimes 1 \cdot(-1)^{\operatorname{deg} a}
$$

for homogeneous $a \in \mathbb{C}^{N \mid N}$. Assignment $a \otimes 1 \mapsto a$ induces an isomorphism of this quotient to $\mathbb{C}^{N \mid N}$. By (5.5), for any $s \geqslant 0$ the generator $T_{i j}^{(s+1)} \in \mathrm{Y}\left(\mathfrak{q}_{N}\right)$ acts on the vector $a \otimes 1 \in \mathbb{C}^{N \mid N} \otimes U_{z}$ as the operator $\left(E_{j i} \otimes z^{s}+E_{-j,-i} \otimes(-z)^{s}\right) \cdot(-1)^{\bar{j}+1}$. Comparing this with the definition (2.21), we complete the proof

Let us use the notion of a $\mathbb{Z}_{2}$-graded irreducibility. When the $\mathbb{Z}_{2}$-graded vector space $U$ is finite-dimensional, the representation $\xi$ in $U$ will be called irreducible if any $\mathbb{Z}_{2}$-graded subspace in $U$ preserved by $\xi$ is either the zero space or $U$ itself. 
Theorem 5.5. Suppose that the representation of the $\mathbb{Z}_{2}$-graded algebra $\mathcal{A}_{n}$ in $U$ is finite-dimensional and irreducible. Then the finite-dimensional representation of the $\mathbb{Z}_{2}$-graded algebra $\mathrm{Y}\left(\mathfrak{q}_{N}\right)$ in $V$ is also irreducible.

Proof. We extend the arguments from [A, Section 4]. The algebra $\mathrm{Y}\left(\mathfrak{q}_{N}\right)$ contains the enveloping algebra $\mathrm{U}\left(\mathfrak{q}_{N}\right)$ as a subalgebra, see (2.13). Representation of this subalgebra in $\left(\mathbb{C}^{N \mid N}\right)^{\otimes n} \otimes U$ is the tensor product of $n$ copies of the defining representation in $\mathbb{C}^{N \mid N}$ and the trivial representation in $U$, see (5.3). Let $V_{0}$ be any non-zero $\mathbb{Z}_{2}$-graded subspace in $V=\mathcal{F}_{N}(U)$, preserved by the action of $\mathrm{Y}\left(\mathfrak{q}_{N}\right)$. In particular, $V_{0}$ is preserved by the action of $\mathrm{U}\left(\mathfrak{q}_{N}\right)$. By [S2, Theorem 3] there is a $\mathbb{Z}_{2}$-graded subspace $U_{0} \subset U$ preserved by $\xi\left(H_{n}\right)$, such that $V_{0} \subset V$ corresponds to $U_{0}$. Assume that for any non-zero vector $b \in U_{0}$ the image in $V_{0}$ of the subspace $\left(\mathbb{C}^{N \mid N}\right)^{\otimes n} \otimes b \subset\left(\mathbb{C}^{N \mid N}\right)^{\otimes n} \otimes U_{0}$ is not zero. Since $\xi$ is irreducible, $\xi\left(\mathcal{A}_{n}\right) \cdot U_{0}=U$. Let us show that the subspace $V_{0} \subset V$ is also $\mathrm{Y}\left(\mathfrak{q}_{N}\right)$-cyclic.

Consider the representation of the algebra $\mathcal{A}_{n}$ induced from the representation of its subalgebra $H_{n}$ in $U_{0} ; \xi$ is a quotient of this induced representation. Thus instead of $\xi$ it suffices to take the induced representation. Realise it in the quotient space $U^{\prime}$ of $\mathcal{A}_{n} \otimes U_{0}$ with respect to the relations $(X Y) \otimes b=X \otimes(\xi(Y) b)$ for all homogeneous $X \in \mathcal{A}_{n}, Y \in H_{n}$ and $b \in U_{0}$. Instead of the subspace $U_{0} \subset U$ it suffices to take the image of the subspace $1 \otimes U_{0} \subset \mathcal{A}_{n} \otimes U_{0}$ in this quotient. Then we have to prove that the subspace in $V^{\prime}=\mathcal{F}_{N}\left(U^{\prime}\right)$ corresponding to the image in $U^{\prime}$ of $1 \otimes U_{0}$, is $\mathrm{Y}\left(\mathfrak{q}_{N}\right)$-cyclic.

There is an ascending $\mathbb{Z}$-filtration on the algebra $\mathcal{A}_{n}$ such that any generator $x_{p}$ is of degree one while $w_{p q}$ and $c_{p}$ are of degree zero, see (5.1). The filtration on $\mathcal{A}_{n}$ induces an ascending $\mathbb{Z}$-filtration on the vector space $\left(\mathbb{C}^{N \mid N}\right)^{\otimes n} \otimes \mathcal{A}_{n} \otimes U_{0}$ and on its quotient $V^{\prime}$. This filtration on $V^{\prime}$ is compatible with the action of the algebra $\mathrm{Y}\left(\mathfrak{q}_{N}\right)$, when it is $\mathbb{Z}$-filtered so that the degree of the generator $T_{i j}^{(s+1)}$ is $s$. But the corresponding $\mathbb{Z}$-graded algebra is isomorphic to $U(\mathfrak{g})$, see Theorem 2.3. The corresponding $\mathbb{Z}$-graded action of $\mathrm{U}(\mathfrak{g})$ can be realised in the space of co-invariants under the action of the group $S_{n} \ltimes \mathbb{Z}_{2}^{n}$ in $\left(\mathbb{C}^{N \mid N}\right)^{\otimes n} \otimes U_{0} \otimes \mathbb{C}\left[x_{1}, \ldots, x_{n}\right]$. Here the action in $\left(\mathbb{C}^{N \mid N}\right)^{\otimes n} \otimes U_{0}$ is determined by $\alpha$ while the action in $\mathbb{C}\left[x_{1}, \ldots, x_{n}\right]$ is standard: any permutation $w \in S_{n}$ acts as $x_{p} \mapsto x_{w(p)}$, the generator of the $q$-th factor $\mathbb{Z}_{2}$ in $\mathbb{Z}_{2}^{n}$ acts as $x_{p} \mapsto(-1)^{\delta_{p q}} x_{p}$. Let $W$ be this space of co-invariants. The $\mathbb{Z}$-graded action of the algebra $\mathrm{U}(\mathfrak{g})$ in $W$ is induced by its action in the space $\left(\mathbb{C}^{N \mid N}\right)^{\otimes n} \otimes U_{0} \otimes \mathbb{C}\left[x_{1}, \ldots, x_{n}\right]$, where the generator $F_{i j}^{(s)} \in \mathfrak{g}$ acts as the operator

$$
\sum_{1 \leqslant p \leqslant n}\left(\iota_{p}\left(E_{i j}\right)+(-1)^{s} \iota_{p}\left(E_{-i,-j}\right)\right) \otimes 1 \otimes x_{p}^{s} .
$$

We have to prove that the subspace $V_{0} \otimes 1 \subset W$ is cyclic under the action of $\mathrm{U}(\mathfrak{g})$.

Let $u_{1}, \ldots, u_{n}$ be complex variables. Let $\varpi_{n}$ be the supersymmetrisation map in the space

$$
\left(\operatorname{End}\left(\mathbb{C}^{N \mid N}\right)[u]\right)^{\otimes n}=\operatorname{End}\left(\mathbb{C}^{N \mid N}\right)^{\otimes n}\left[u_{1}, \ldots, u_{n}\right],
$$

as in the proof of Proposition 2.2. We have normalised this map so that $\varpi_{n}^{2}=\varpi_{n}$. Consider the homomorphism $\mathrm{U}(\mathfrak{g}) \rightarrow \operatorname{End}\left(\mathbb{C}^{N \mid N}\right)^{\otimes n}\left[u_{1}, \ldots, u_{n}\right]$ determined by

$$
F_{i j}^{(s)} \mapsto \sum_{1 \leqslant p \leqslant n}\left(\iota_{p}\left(E_{i j}\right)+(-1)^{s} \iota_{p}\left(E_{-i,-j}\right)\right) u_{p}^{s}
$$


Image of $\mathrm{U}(\mathfrak{g})$ under this homomorphism consists of all polynomials $F\left(u_{1}, \ldots, u_{n}\right)$ valued in $\operatorname{End}\left(\mathbb{C}^{N \mid N}\right)^{\otimes n}$ which are $\varpi_{n}$-invariant and for each $p=1, \ldots, n$ satisfy

$$
\left(\mathrm{id}^{\otimes(p-1)} \otimes \eta \otimes \mathrm{id}^{\otimes(n-p)}\right) F\left(u_{1}, \ldots, u_{n}\right)=F\left(u_{1}, \ldots,-u_{p}, \ldots, u_{n}\right) .
$$

This follows from the Poincaré-Birkhoff-Witt theorem for Lie superalgebras.

Now consider the subspace in $\left(\mathbb{C}^{N \mid N}\right)^{\otimes n} \otimes U_{0} \otimes \mathbb{C}\left[x_{1}, \ldots, x_{n}\right]$ consisting of all invariants under the action of the group $S_{n} \ltimes \mathbb{Z}_{2}^{n}$. Denote by $W_{*}$ this subspace. Also consider the subspace $W_{0}$ in the tensor product $\left(\mathbb{C}^{N \mid N}\right)^{\otimes n} \otimes U_{0}$ consisting of all $\alpha$-invariants. We shall prove that the subspace $W_{0}$ is cyclic under the action of the algebra $\operatorname{End}\left(\mathbb{C}^{N \mid N}\right)^{\otimes n}$ in the first tensor factor. The above description of the image of $\mathrm{U}(\mathfrak{g})$ under (5.7) will then imply, that the subspace $W_{0} \otimes 1 \subset W_{*}$ is $\mathrm{U}(\mathfrak{g})$-cyclic. But this will yield $\mathrm{U}(\mathfrak{g})$-cyclicity of the subspace $V_{0} \otimes 1 \subset W$.

Take any $\alpha$-invariant inner product $\langle$,$\rangle on the vector space \left(\mathbb{C}^{N \mid N}\right)^{\otimes n} \otimes U_{0}$. Now suppose that the subspace $W_{0} \subset\left(\mathbb{C}^{N \mid N}\right)^{\otimes n} \otimes U_{0}$ is not $\operatorname{End}\left(\mathbb{C}^{N \mid N}\right)^{\otimes n}$-cyclic. Then we have $\left\langle\left(\mathbb{C}^{N \mid N}\right)^{\otimes n} \otimes b, W_{0}\right\rangle=\{0\}$ for some non-zero vector $b \in U_{0}$. But this contradicts to our initial choice of the subspace $U_{0} \subset U$

A method for constructing the irreducible finite-dimensional representations of the algebra $\mathcal{A}_{n}$ was developed in [N2]. Restriction of any such representation $U$ to the subalgebra $H_{n} \subset \mathcal{A}_{n}$ is a quotient of the left regular representation of $H_{n}$. The restriction of the corresponding representation $V$ of $\mathrm{Y}\left(\mathfrak{q}_{N}\right)$ to the subalgebra $\mathrm{U}\left(\mathfrak{q}_{N}\right)$ is then a quotient of the representation of $\mathrm{U}\left(\mathfrak{q}_{N}\right)$ in $\left(\mathbb{C}^{N \mid N}\right)^{\otimes n}$. But there are irreducible finite-dimensional representations of the algebra $\mathrm{U}\left(\mathfrak{q}_{N}\right)$, which do not appear as quotients of the representation in $\left(\mathbb{C}^{N \mid N}\right)^{\otimes n}$ for any $n$, see $[\mathrm{P}]$. Thus our correspondence $U \mapsto V$ cannot provide all irreducible representations of the algebra $\mathrm{Y}\left(\mathfrak{q}_{N}\right)$. It would be interesting to give a parametrisation of all irreducible finitedimensional representations of the algebra $\mathrm{Y}\left(\mathfrak{q}_{N}\right)$; $\mathrm{cf}$. [D4, Theorem 2] and $[\mathrm{M}]$.

\section{Acknowledgements}

I am grateful to I. Cherednik, G. Olshanski and A. Sudbery for helpful discussions. I am also grateful to V. Drinfeld, P. Kulish and E. Sklyanin for their kind interest

in this work. Support from the EPSRC by an Advanced Research Fellowship, and from the EC under the TMR grant FMRX-CT97-0100, is gratefully acknowledged.

\section{References}

[A] T. Arakawa, Drinfeld functor and finite-dimensional representations of the Yangian, Commun. Math. Phys. (1999), math/9807144.

[A1] J. Avan, Graded Lie algebras in the Yang-Baxter equation, Phys. Lett. B 245 (1990), 491-496.

[A2] J. Avan, Current algebra realization of $R$-matrices associated to $Z_{2}$-graded Lie algebras, Phys. Lett. B 252 (1990), 230-236.

[BD] A. Belavin and V. Drinfeld, The classical Yang-Baxter equation for simple Lie algebras, Funct. Anal. Appl. 17 (1983), 220-221.

[BL] D. Bernard and A. LeClair, The quantum double in integrable quantum field theories, Nucl. Phys. B 399 (1993), 709-748.

[BGHP] D. Bernard, M. Gaudin, F. Haldane and V. Pasquier, Yang-Baxter equation in long-range interacting systems, J. Phys. A 26 (1993), 5219-5236. 
[C] A. Capelli, Sur les opérations dans la théorie des formes algébriques, Math. Ann. 37 (1890), 1-37.

[D1] V.Drinfeld, Hopf algebras and the quantum Yang-Baxter equation, Soviet Math. Dokl. 32 (1985), 254-258.

[D2] V. Drinfeld, Degenerate affine Hecke algebras and Yangians, Funct. Anal. Appl. 20 (1986), 56-58.

[D3] V. Drinfeld, Quantum groups, International Congress of Mathematicians 1986, Amer. Math. Soc., Providence, 1987, pp. 798-820.

[D4] V. Drinfeld, A new realization of Yangians and quantized affine algebras, Soviet Math. Dokl. 36 (1988), 212-216.

[FR] L. Faddeev and N. Reshetikhin, Hamiltonian structures for integrable field theory models, Theoret. Math. Phys. 56 (1983), 847-862.

[JN] A. Jones and M. Nazarov, Affine Sergeev algebra and q-analogues of the Young symmetrizers for projective representations of the symmetric group, J. London Math. Soc. 78 (1999), q-alg/9712041.

[K] V. Kac, Lie superalgebras, Adv. in Math. 26 (1977), 8-96.

[KT] S. Khoroshkin and V. Tolstoy, Yangian double, Lett. Math. Phys. 36 (1996), 373-402.

[LS] D. Leites and V. Serganova, Solutions of the classical Yang-Baxter equation for simple Lie superalgebras, Theoret. Math. Phys. 58 (1984), 16-24.

[M] A. Molev, Finite-dimensional irreducible representations of twisted Yangians, J. Math. Phys. 39 (1998), 5559-5600.

[MM] J. Milnor and J. Moore, On the structure of Hopf algebras, Ann. of Math. 81 (1965), 211-264.

[MNO] A. Molev, M. Nazarov and G. Olshanski, Yangians and classical Lie algebras, Russian Math. Surveys 51 (1996), 205-282.

[N1] M. Nazarov, Quantum Berezinian and the classical Capelli identity, Lett. Math. Phys. 21 (1991), 123-131.

[N2] M. Nazarov, Young's symmetrizers for projective representations of the symmetric group, Adv. in Math. 127 (1997), 190-257.

[N3] M. Nazarov, Capelli identities for Lie superalgebras, Ann. Scient.Éc. Norm. Sup. 30 (1997), 847-872.

[N4] M. Nazarov, Yangians and Capelli identities, Amer. Math. Soc. Transl. 181 (1998), 139-163.

[O1] G. Olshanski, Representations of infinite-dimensional classical groups, limits of enveloping algebras, and Yangians, Adv. in Soviet Math. 2 (1991), 1-66.

[O2] G. Olshanski, Quantized universal enveloping superalgebra of type $Q$ and a super-extension of the Hecke algebra, Lett. Math. Phys. 24 (1992), 93-102.

$[\mathrm{P}]$ I. Penkov, Characters of typical irreducible finite-dimensional $\mathfrak{q}(n)$-modules, Funct. Anal. Appl. 20 (1986), 30-37.

[RTF] N. Reshetikhin, L. Takhtajan and L. Faddeev, Quantization of Lie groups and Lie algebras, Leningrad Math. J. 1 (1990), 193-225.

[S] F. Smirnov, Dynamical symmetries of massive integrable models, Internat. J. Modern. Phys. A7 (1992), Suppl. 1B, 813-858.

[S1] A. Sergeev, The centre of enveloping algebra for Lie superalgebra $Q(n, \mathbb{C})$, Lett. Math. Phys. 7 (1983), 177-179.

[S2] A. Sergeev, The tensor algebra of the identity representation as a module over the Lie superalgebras $G L(n, m)$ and $Q(n)$, Math. Sbornik 51 (1985), 419-427. 